\title{
The Future of Particle Physics
}

Chris Quigg

Fermi National Accelerator Laboratory

Physikalisches Kolloquium · Universität Heidelberg • 20.X.20I7 


\section{Three Cheers for Multimessenger Astronomy!}

\begin{tabular}{|c|c|c|}
\hline PRL 119, 161101 (2017) & $\begin{array}{l}\text { Plel Selected for a Viewpoint in Physics } \\
\text { PHYSICAL REVIEW LETTERS }\end{array}$ & $\begin{array}{l}\text { week ending } \\
20 \text { OCTOBER } 2017\end{array}$ \\
\hline
\end{tabular}

GW170817: Observation of Gravitational Waves from a Binary Neutron Star Inspiral

$$
\text { B. P. Abbott et al. }
$$

(LIGO Scientific Collaboration and Virgo Collaboration)

(Received 26 September 2017; revised manuscript received 2 October 2017; published 16 October 2017)

On August 17, 2017 at 12:41:04 UTC the Advanced LIGO and Advanced Virgo gravitational-wave

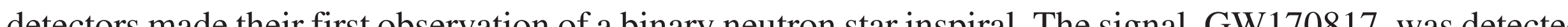
detecto me $8.0 \times 10^{4}$ yeas. We infer the compont 32.4 a a $8.0 \times 10$ years. We infer the component masses of the binary to be between 0.86 and $2.26 M_{\odot}$, in agreement with masses of known neutron stars. Restricting the component spins to the range inferred in binary neutron stars, we find the component masses to be in the range 1.17-1.60 $M_{\odot}$, with the total mass of the system $2.74_{-0.01}^{+0.04} M_{\odot}$. The source was localized within a sky region of $28 \mathrm{deg}^{2}(90 \%$ probability) and had a luminosity distance of $40_{-14}^{+8} \mathrm{Mpc}$, the closest and most precisely localized gravitational-wave signa yet. The association with the $\gamma$-ray burt GRB 170817A, detected by Fermi-GBM 1.7 s after the yet.

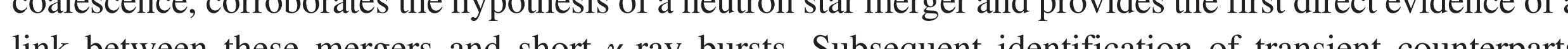
link be across the electromagnetic spectrum in the same location further supports the interpretation of this event as a neutron star merger. This unprecedented joint gravitational and electromagnetic observation provides insight into astrophysics, dense matter, gravitation, and cosmology.

DOI: 10.1103/PhysRevLett.119.161101

GW + prompt short GRB, EM transients: test gravity theories, $H_{0}$ determination, heavy-element production (no UHE CRs, v)
400 . LIGO - Virgo

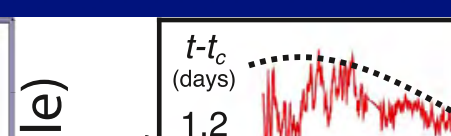
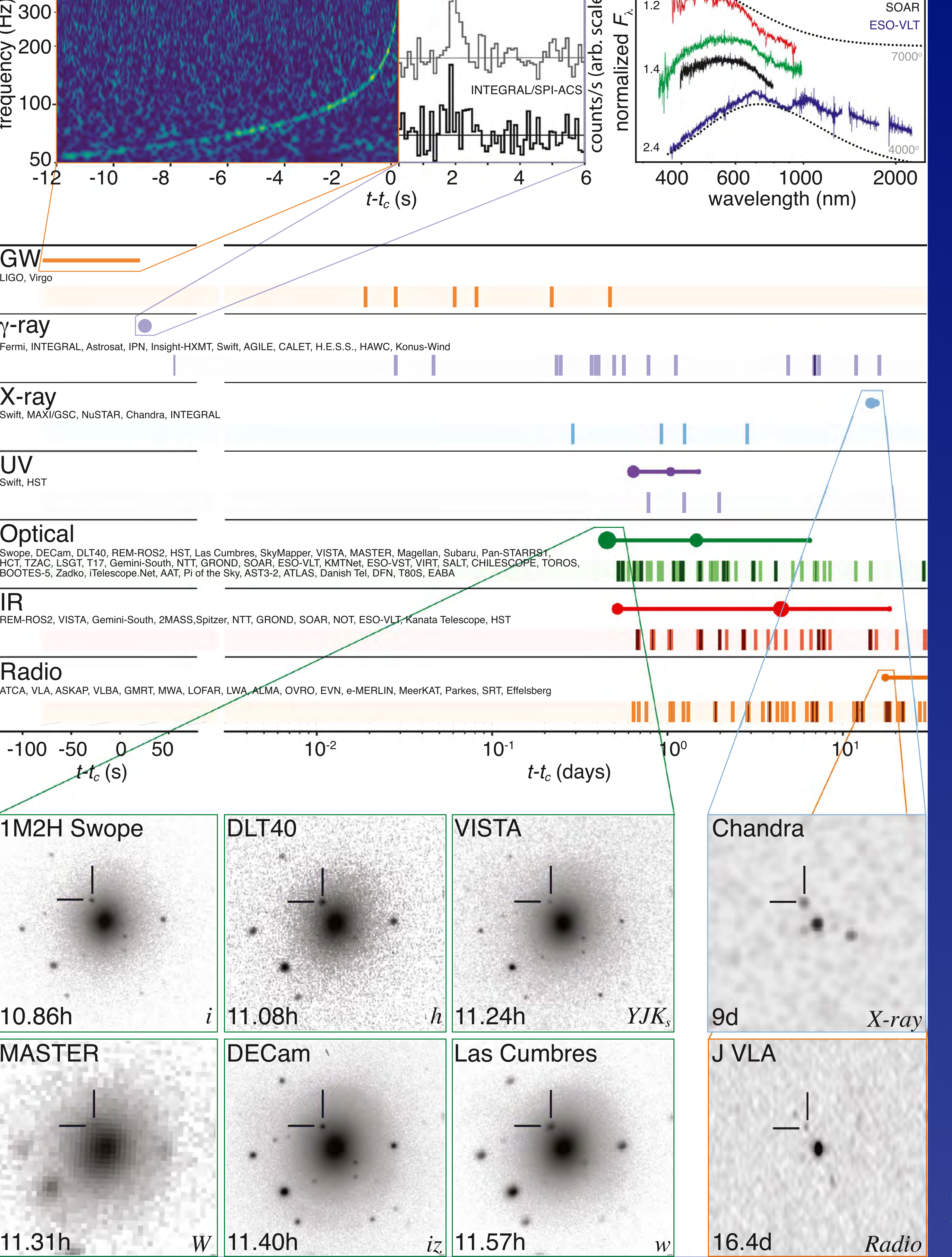


\section{0 years ago: How little we knew}




\section{Problems of High-Energy Physics (NAL Design Report, January 1968)}

We would like to have answers to many questions. Among them are the following:

Which, if any, of the particles that have so far been discovered, is, in fact, elementary, and is there any validity in the concept of "elementary" particles?

What new particles can be made at energies that have not yet been reached? Is there some set of building blocks that is still more fundamental than the neutron and the proton?

Is there a law that correctly predicts the existence and nature of all the particles, and if so, what is that law?

Will the characteristics of some of the very short-lived particles appear to be different when they are produced at such higher velocities that they no longer spend their entire lives within the strong influence of the particle from which they are produced?

Do new symmetries appear or old ones disappear for high momentum-transfer events?

What is the connection, if any, of electromagnetism and strong interactions?
Do the laws of electromagnetic radiation, which are now known to hold over an enormous range of lengths and frequencies, continue to hold in the wavelength domain characteristic of the subnuclear particles?

What is the connection between the weak interaction that is associated with the massless neutrino and the strong one that acts between neutron and proton?

Is there some new particle underlying the action of the "weak" forces, just as, in the case of the nuclear force, there are mesons, and, in the case of the electromagnetic force, there are photons? If there is not, why not?

In more technical terms: Is local field theory valid? A failure in locality may imply a failure in our concept of space. What are the fields relevant to a correct local field theory? What are the form factors of the particles? What exactly is the explanation of the electromagnetic mass difference? Do "weak" interactions become strong at sufficiently small distances? Is the Pomeranchuk theorem true? Do the total cross sections become constant at high energy? Will new symmetries appear, or old ones disappear, at higher energy? 


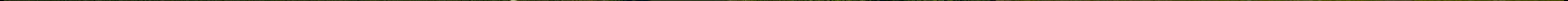




\section{Next for Fermilab: $\underline{\text { CMS }}, \mathrm{g}-2, \mu 2 \mathrm{e}$, DUNE, astroparticle}
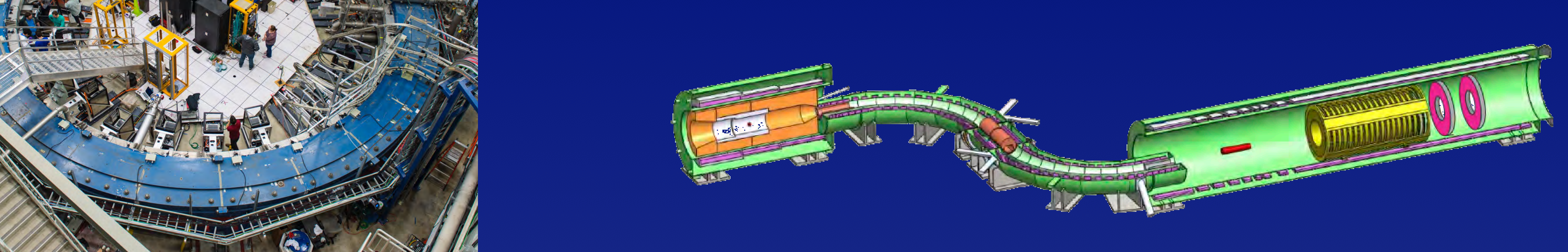

Sanford Underground

Research Facility

\section{DEEP UNDERGROUND}

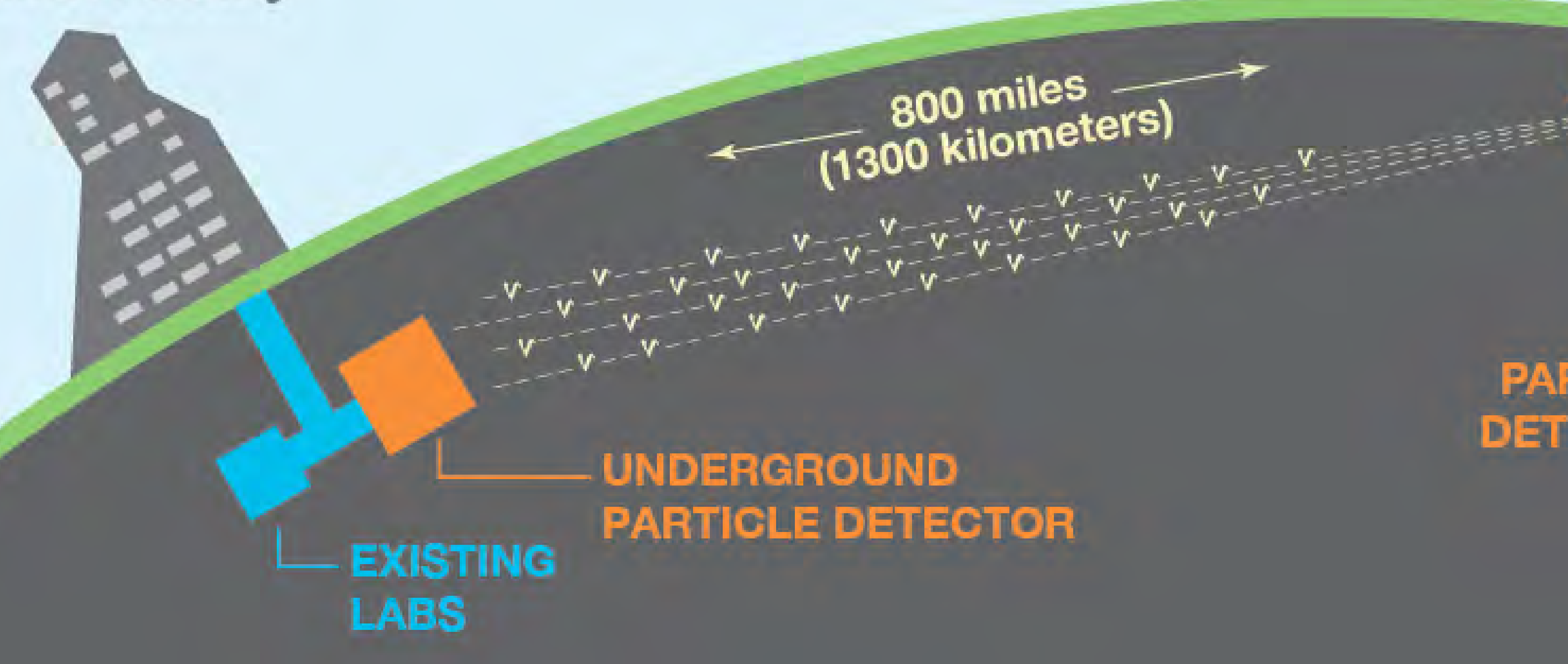

NEUTRINO PRODUCTION

PARTIGLE DETECTOR LABS

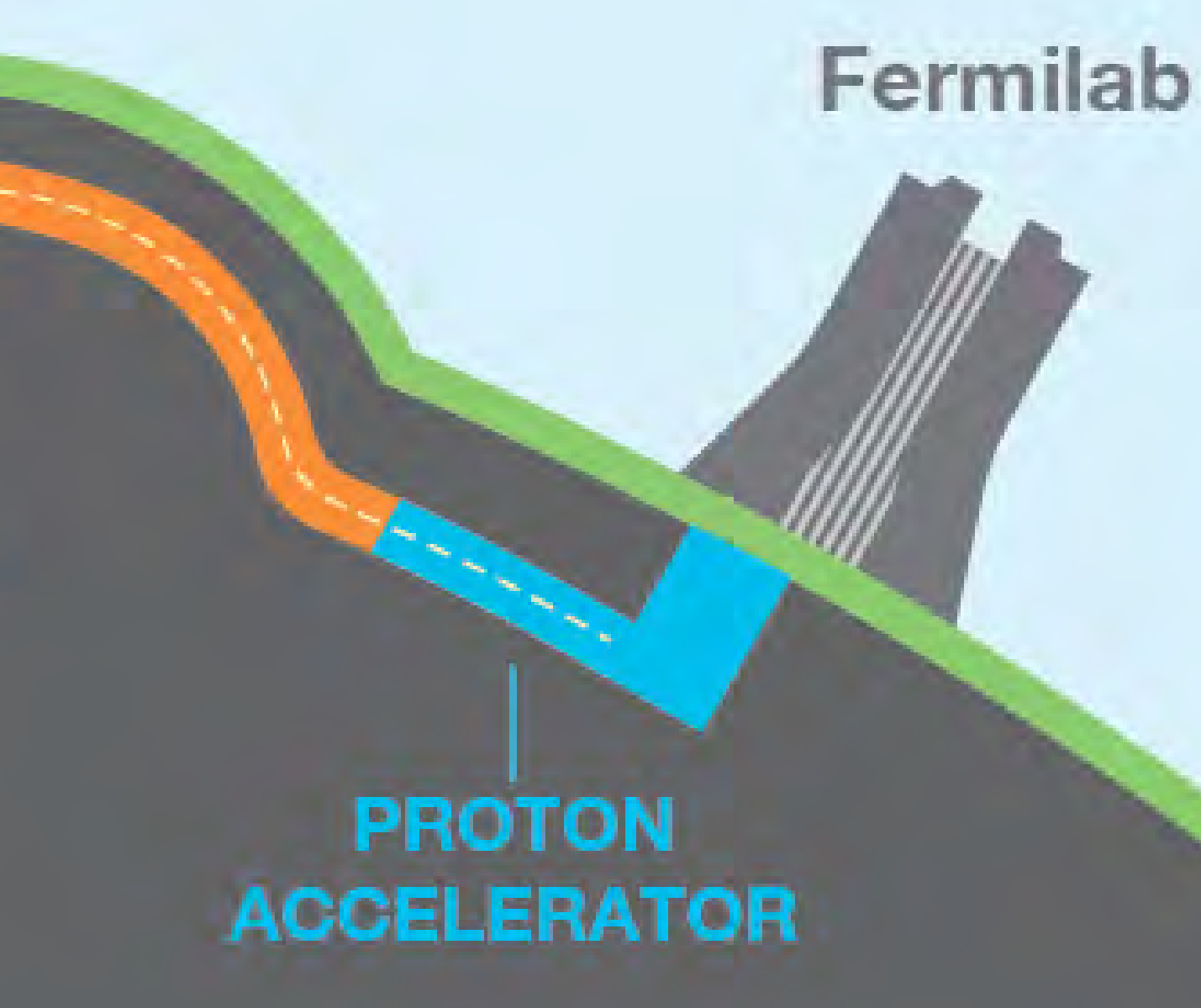




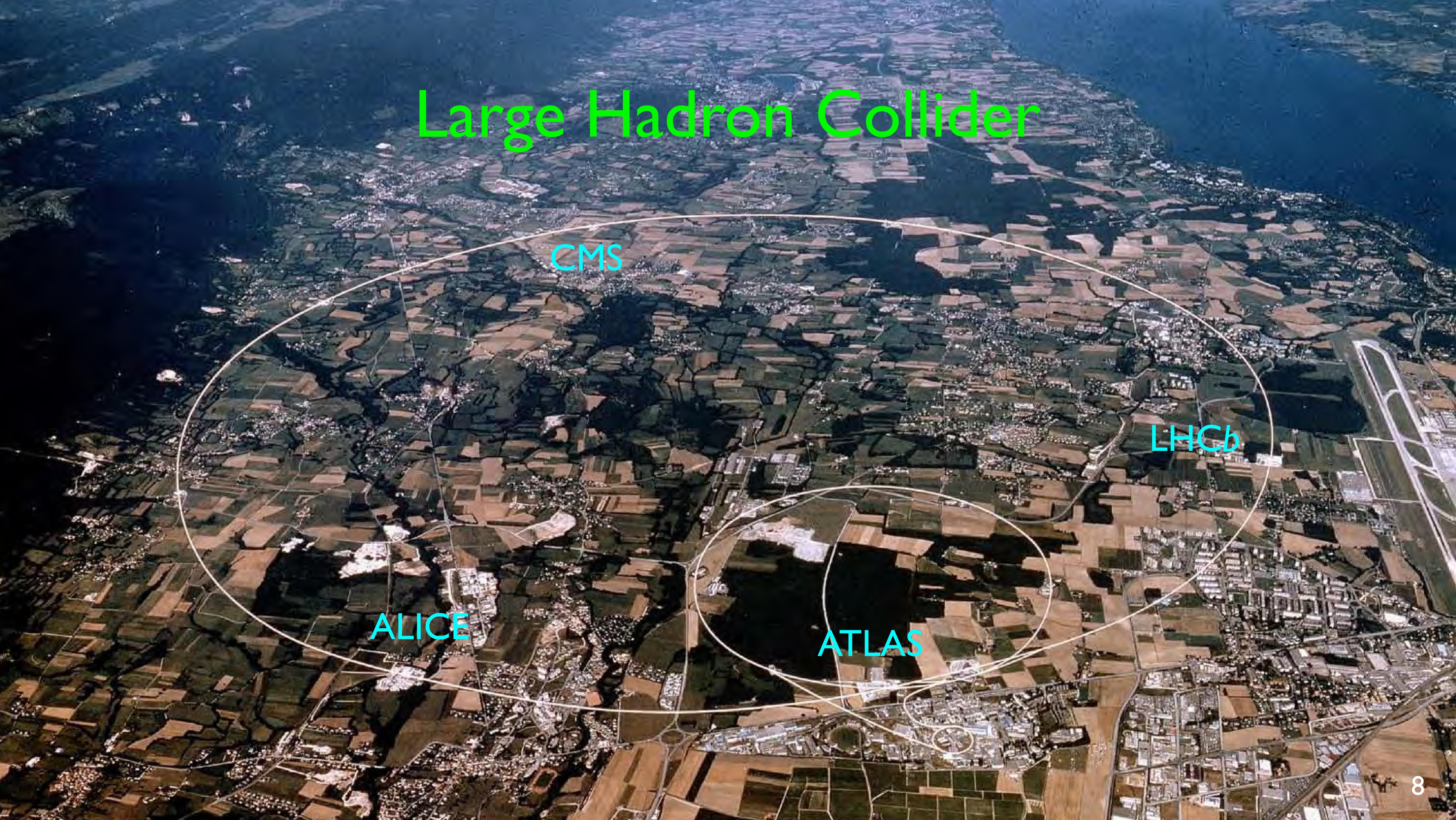




\section{Very-High-Rate Experiments}

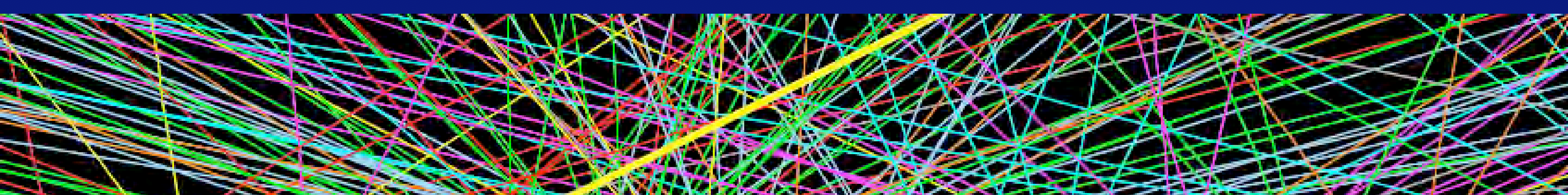

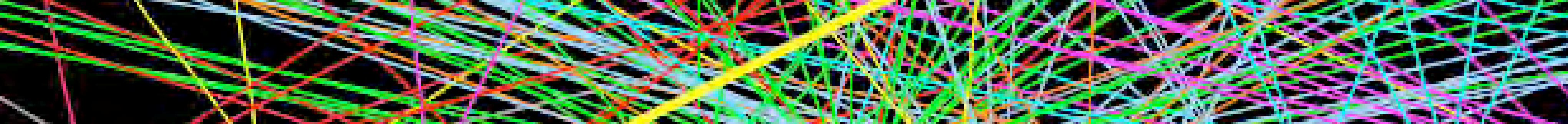
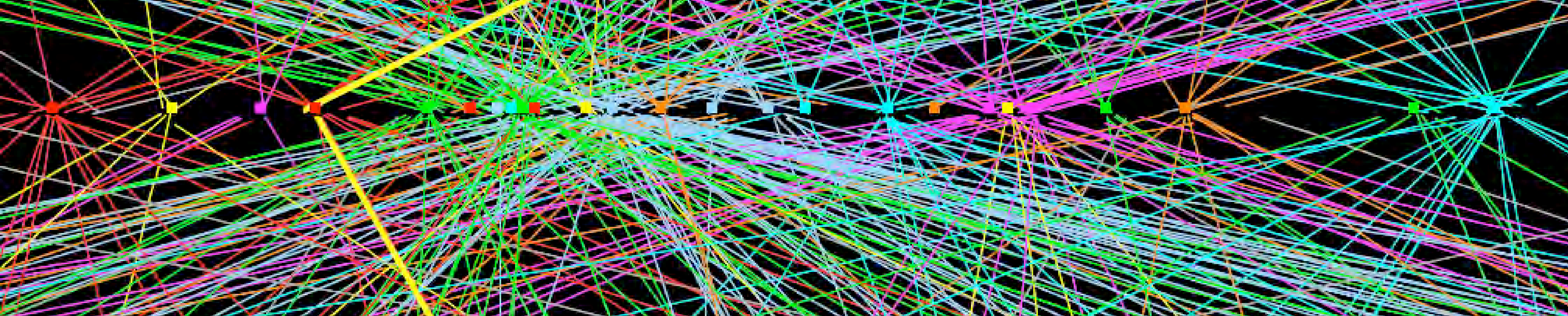

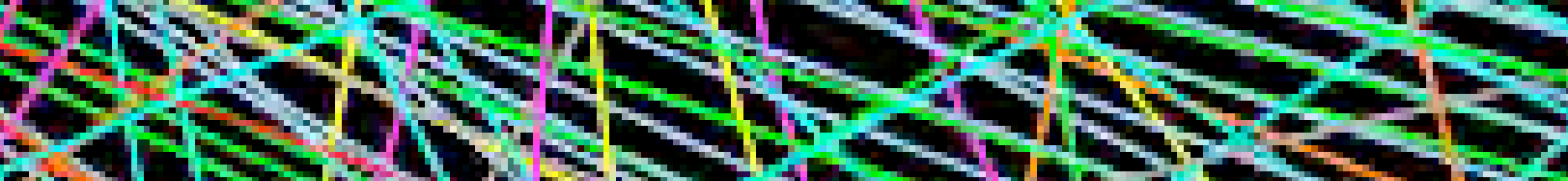

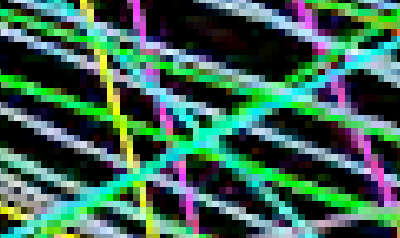

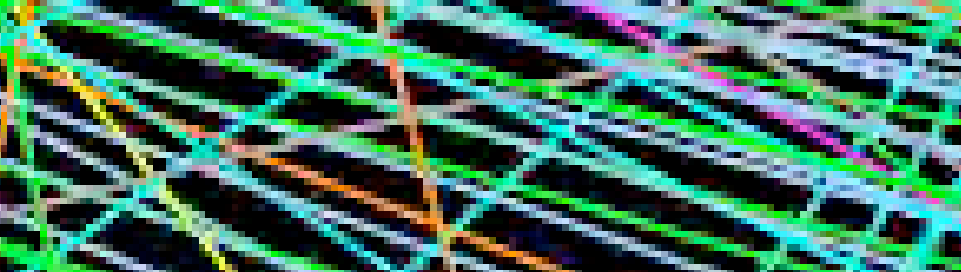

The Allure of Ultrasensitive Experiments

Fermilab Academic Lectures 


\section{Standard Model Production Cross Section Measurements}

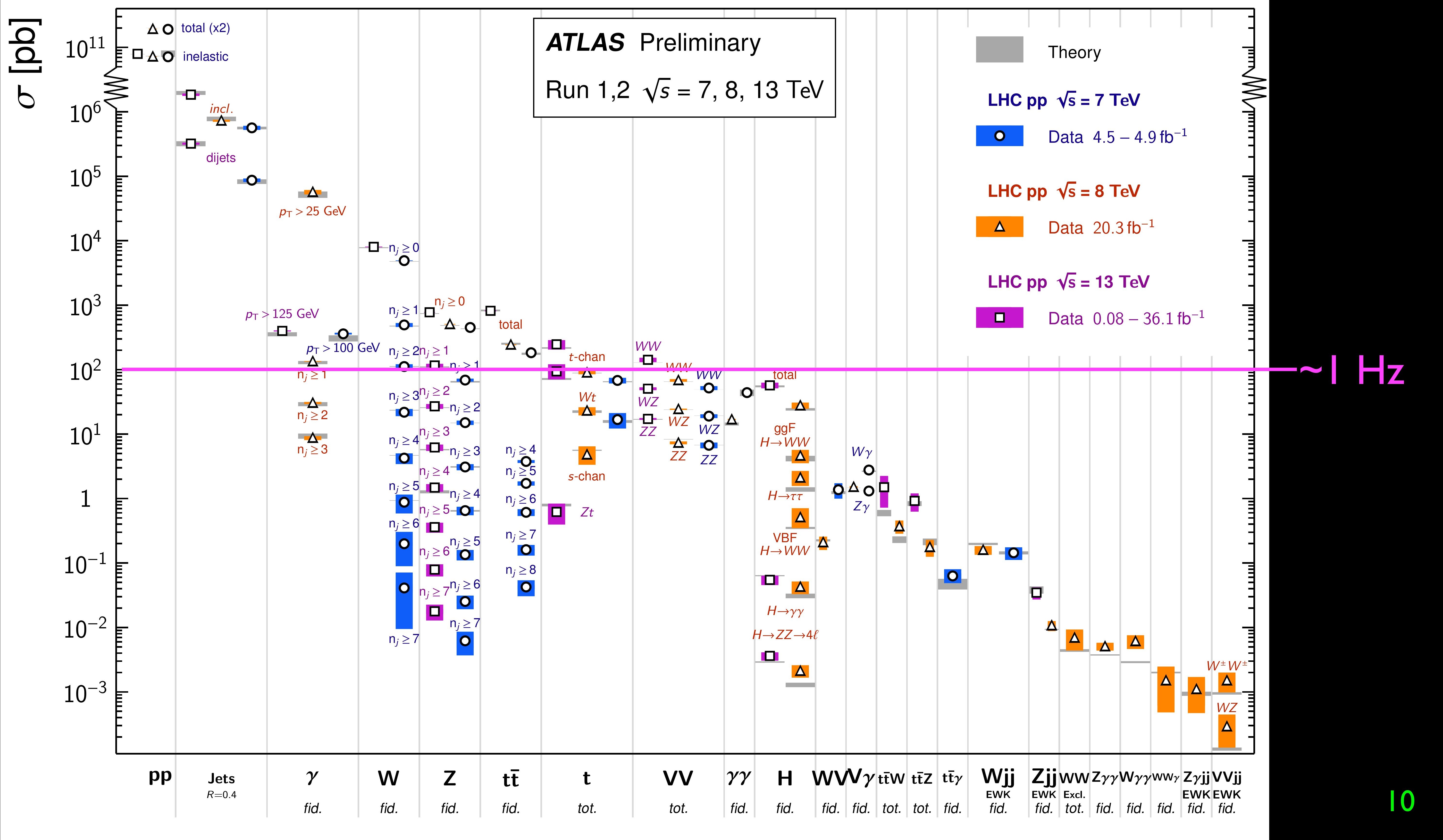




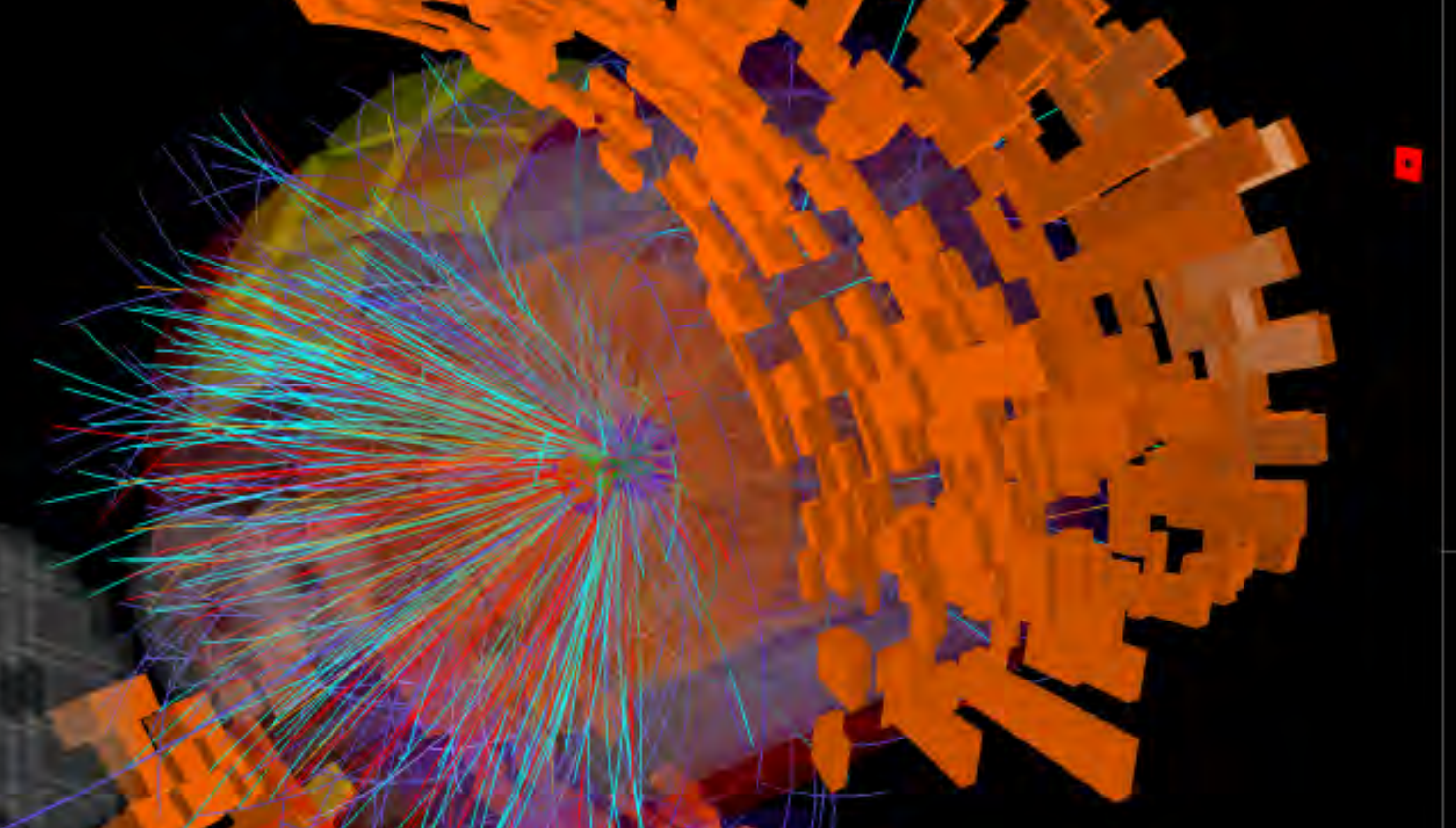

$x_{2} y+x=$

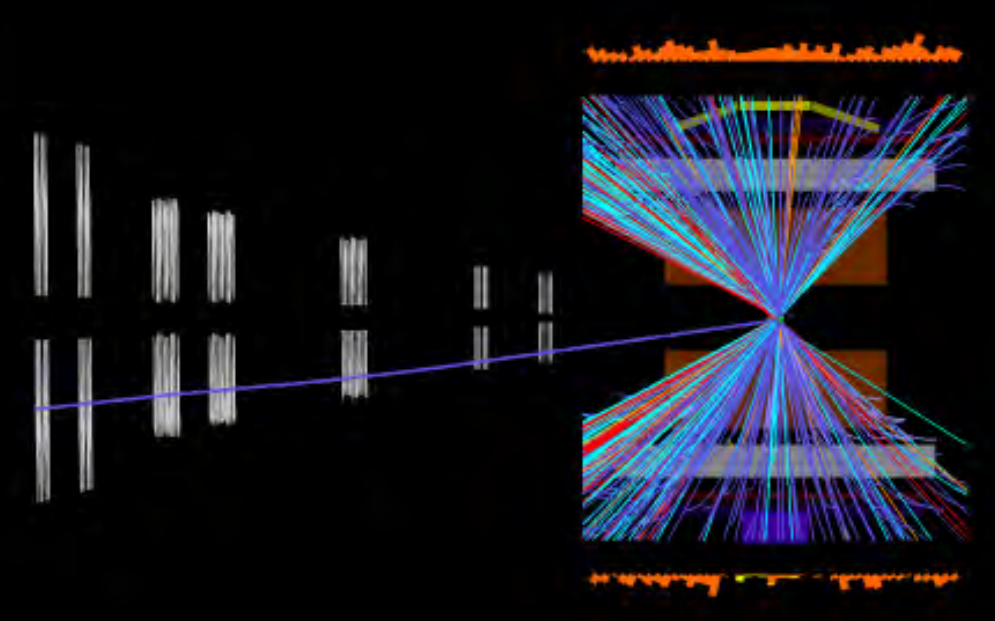

\section{Event 23965322 \\ Run 200429 \\ Fri, 13 Oct 2017 03:26:47}

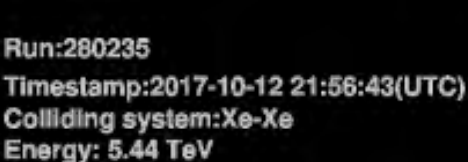

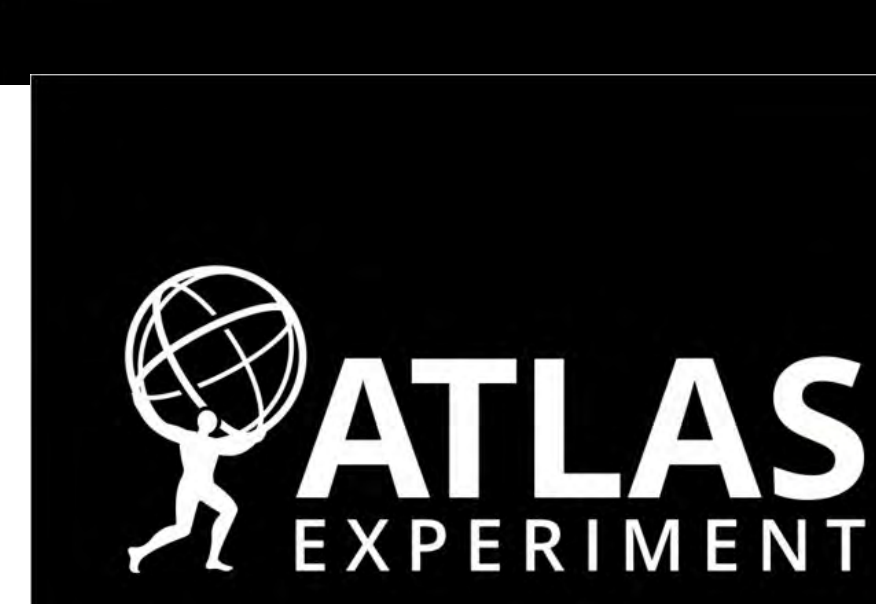

Date: 2017-10-13 01:45:04 CEST

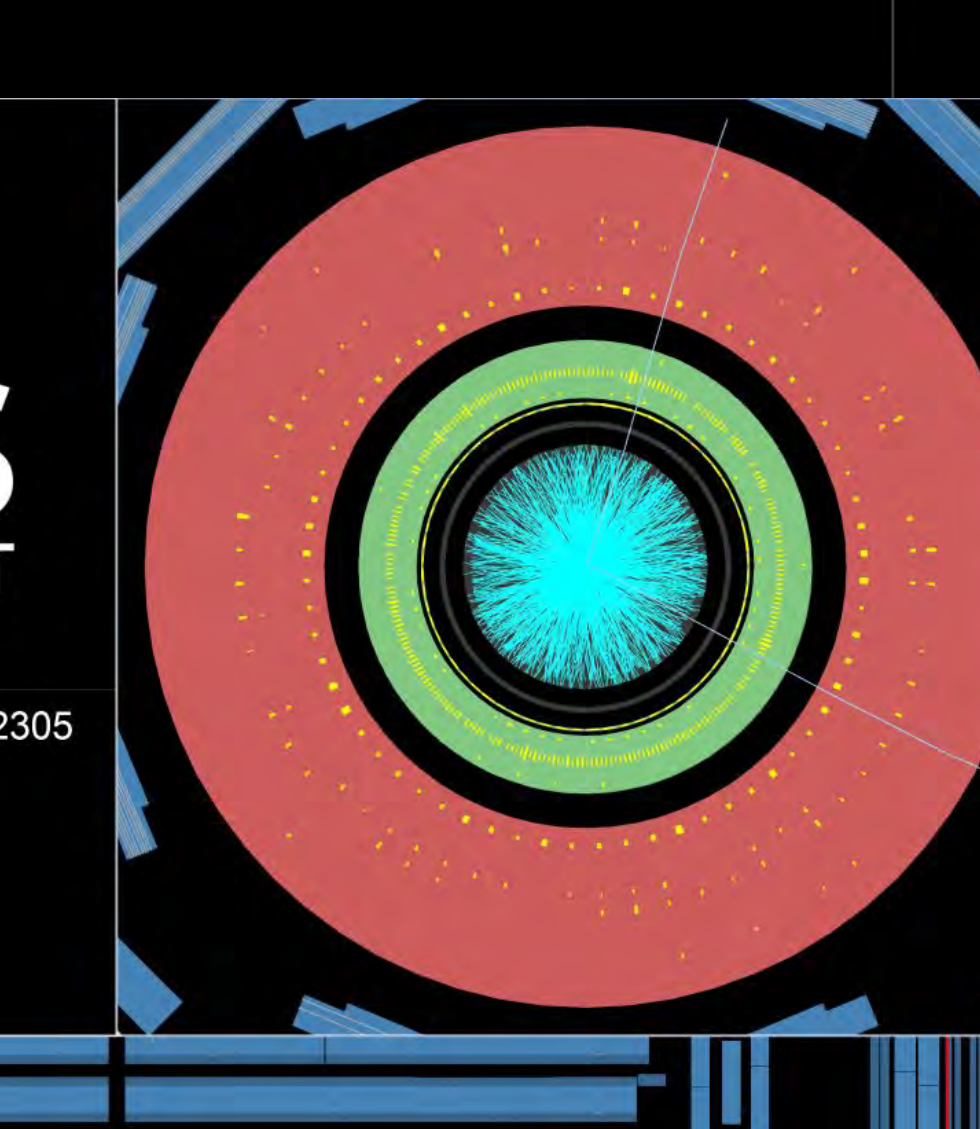

T)
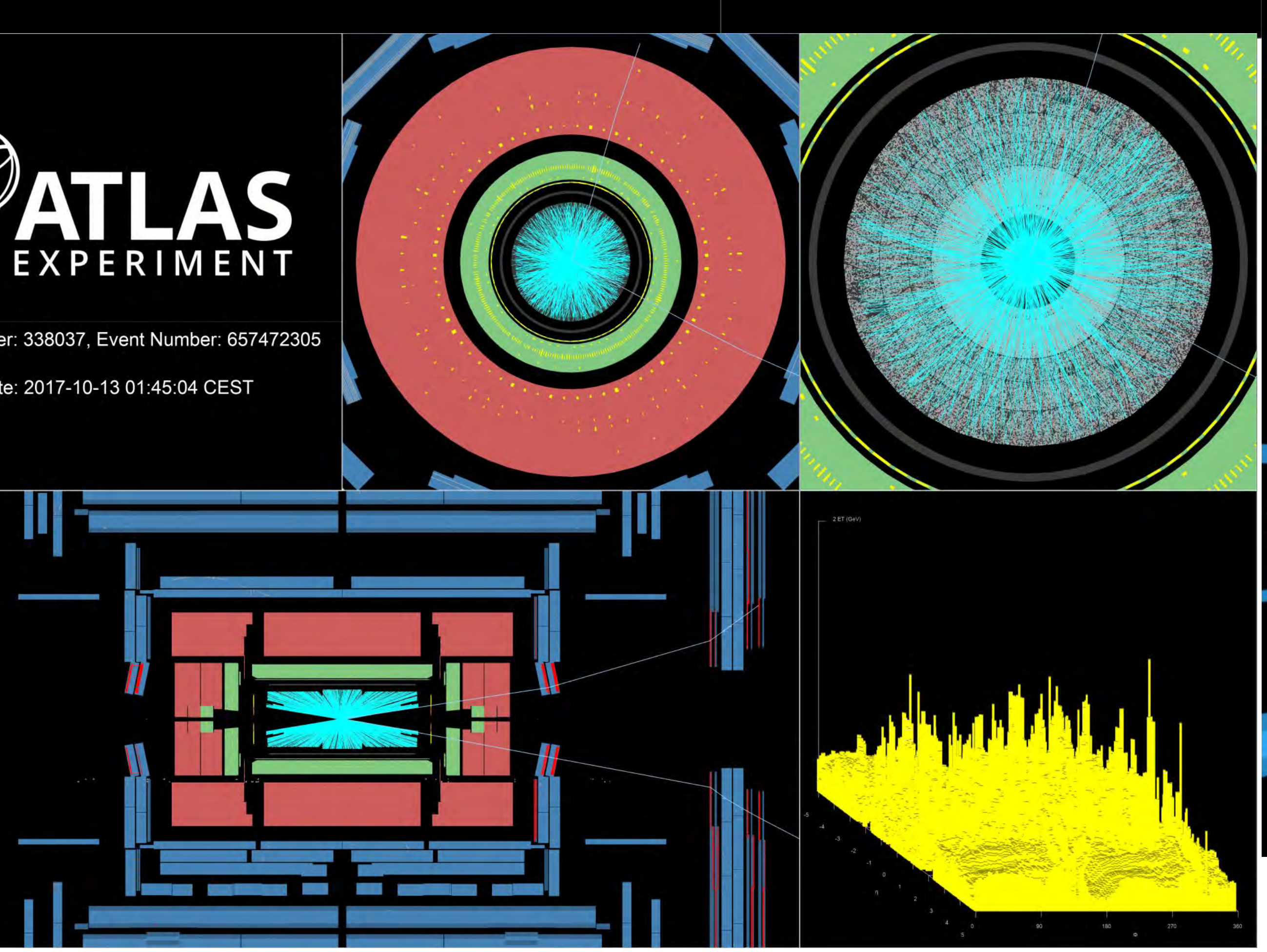

CMS Experiment at the LFC, CERN
Data recorded: 2017-Oct-12 20:44:56,751360 GMt Run / Event / LS: 304899 / 8743361 / 90 "
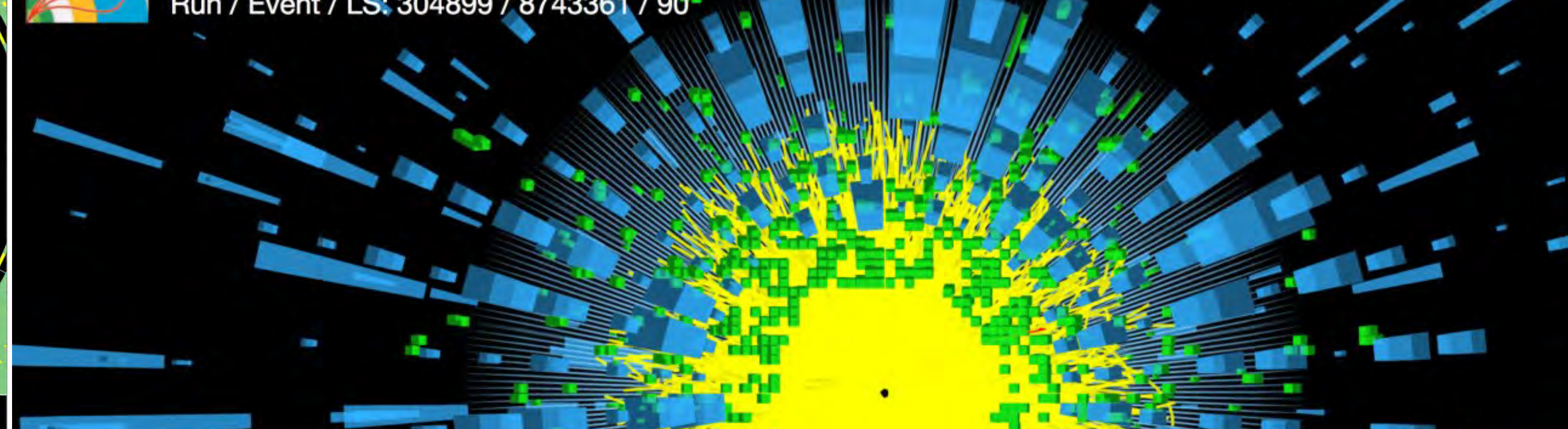

- 4 -

. C .

- 


\section{In a decade or two, we can hope to ...}

Understand electroweak symmetry breaking Observe the Higgs boson

Measure neutrino masses and mixings

Establish Majorana neutrinos $\left(\beta \beta_{0 \nu}\right)$

Thoroughly study $C P$ violation in $B$ decay

Exploit rare decays $(K, D, \ldots)$

Observe $n$ EDM, pursue $e^{-}$EDM

Use top as a tool

Observe new phases of matter

Understand hadron structure quantitatively

Uncover QCD's full implications

Observe proton decay

Understand the baryon excess

Catalogue matter \& energy of universe

Measure dark energy equation of state

Search for new macroscopic forces

Determine GUT symmetry

... learn the right questions to ask
Detect neutrinos from the universe Learn how to quantize gravity

Learn why empty space is nearly weightless

Test the inflation hypothesis

Understand discrete symmetry violation

Resolve the hierarchy problem

Discover new gauge forces

Directly detect dark-matter particles

Explore extra spatial dimensions

Understand origin of large-scale structure

Observe gravitational radiation

Solve the strong $\mathrm{CP}$ problem

Learn whether supersymmetry is TeV-scale

Seek TeV dynamical symmetry breaking

Search for new strong dynamics

Explain the highest-energy cosmic rays

Formulate problem of identity

$\ldots$ and rewrite the textbooks! 


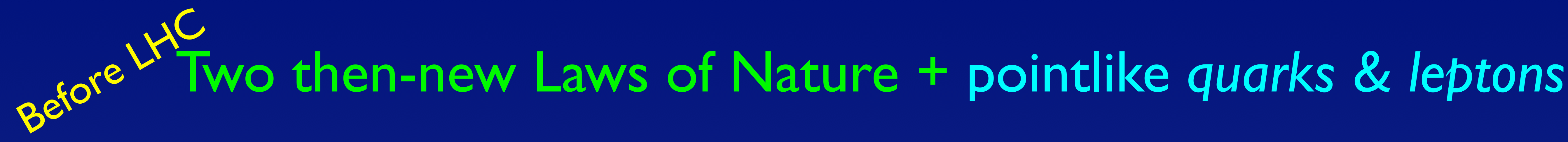

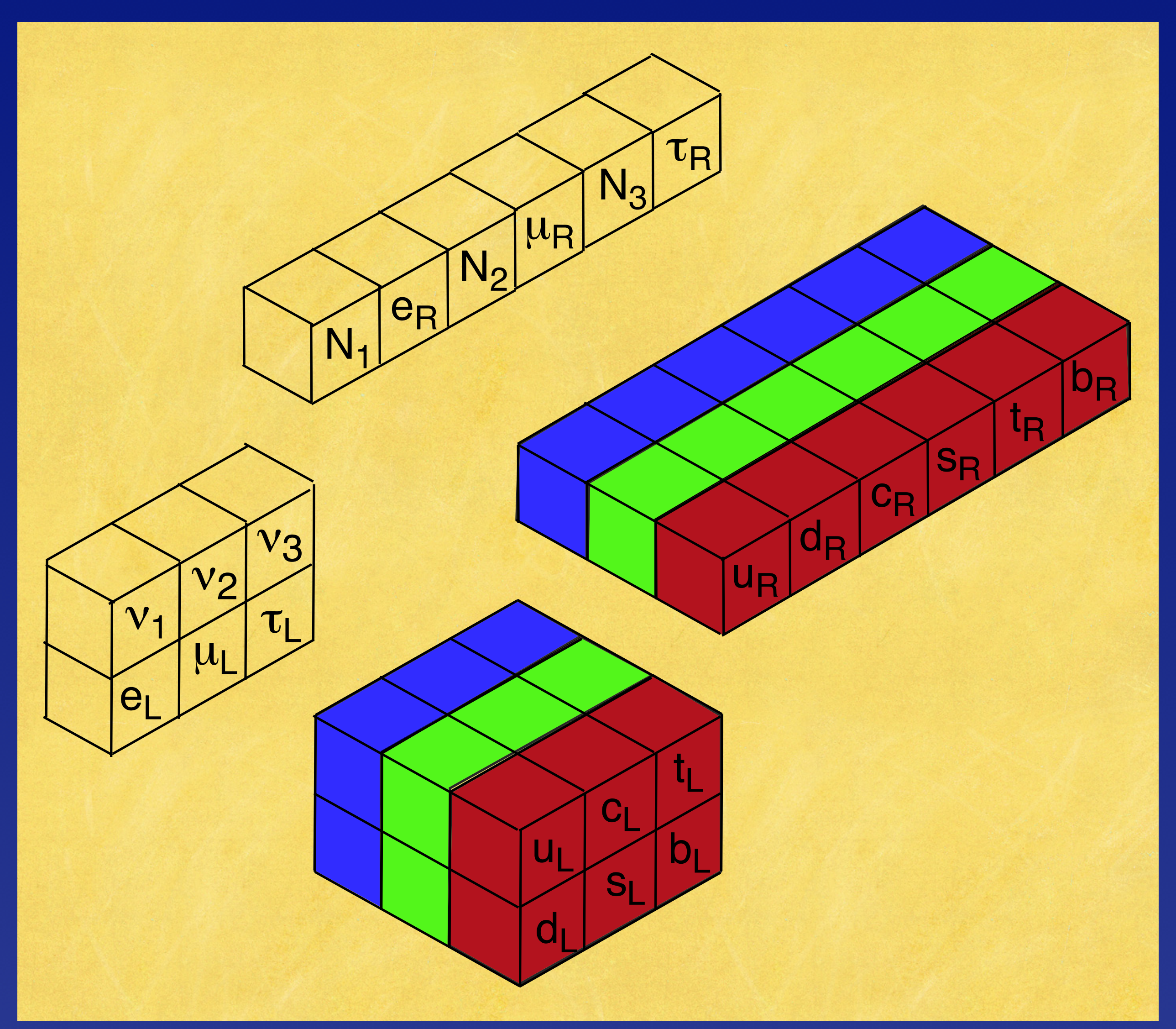

Interactions: $\mathrm{SU}(3)_{c} \otimes S U(2)_{L} \otimes U(1)_{Y}$ gauge symmetries 
Antiscreening evolution of the strong coupling "constant"

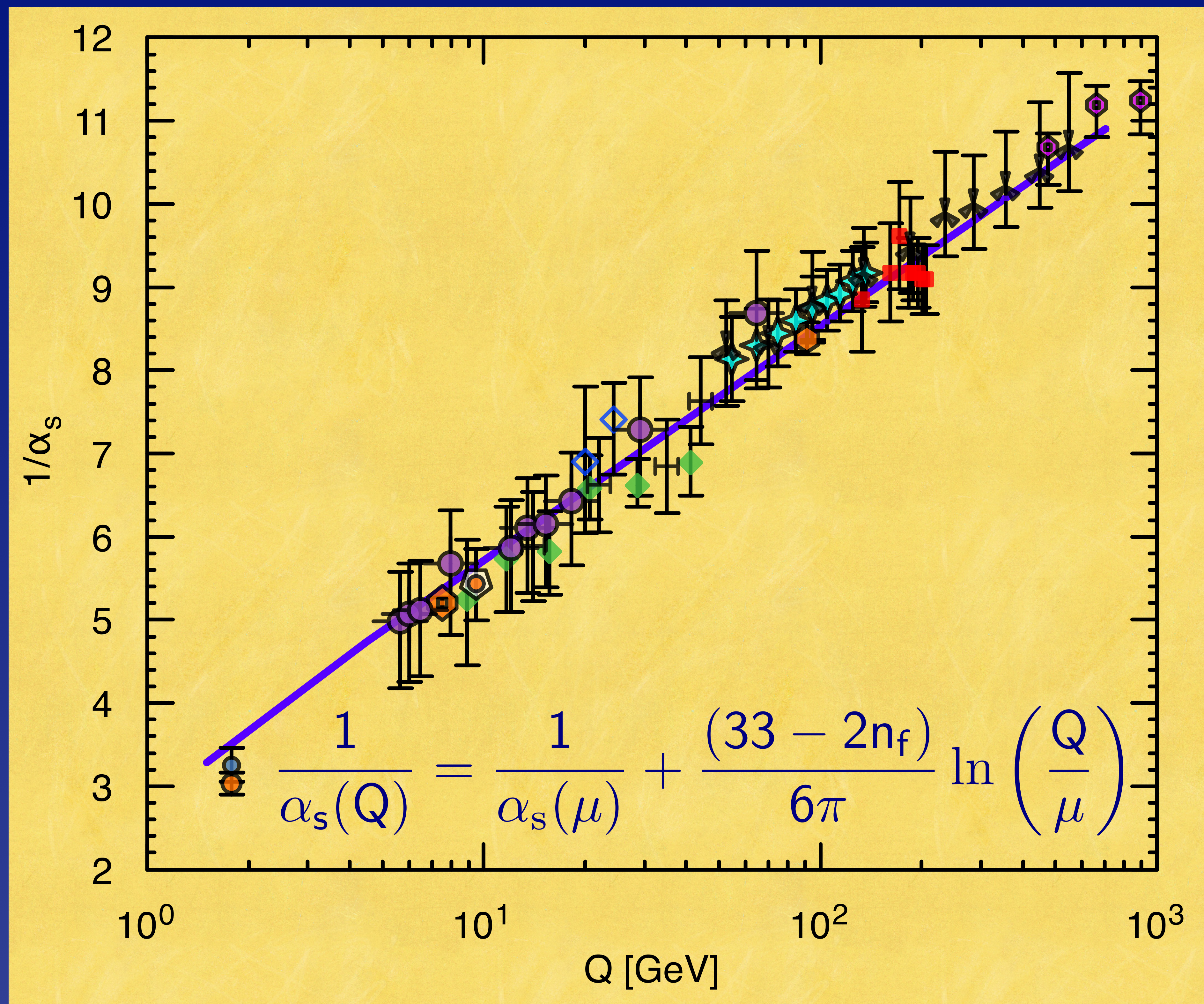


The World's Most Powerful Microscopes nanonanophysics

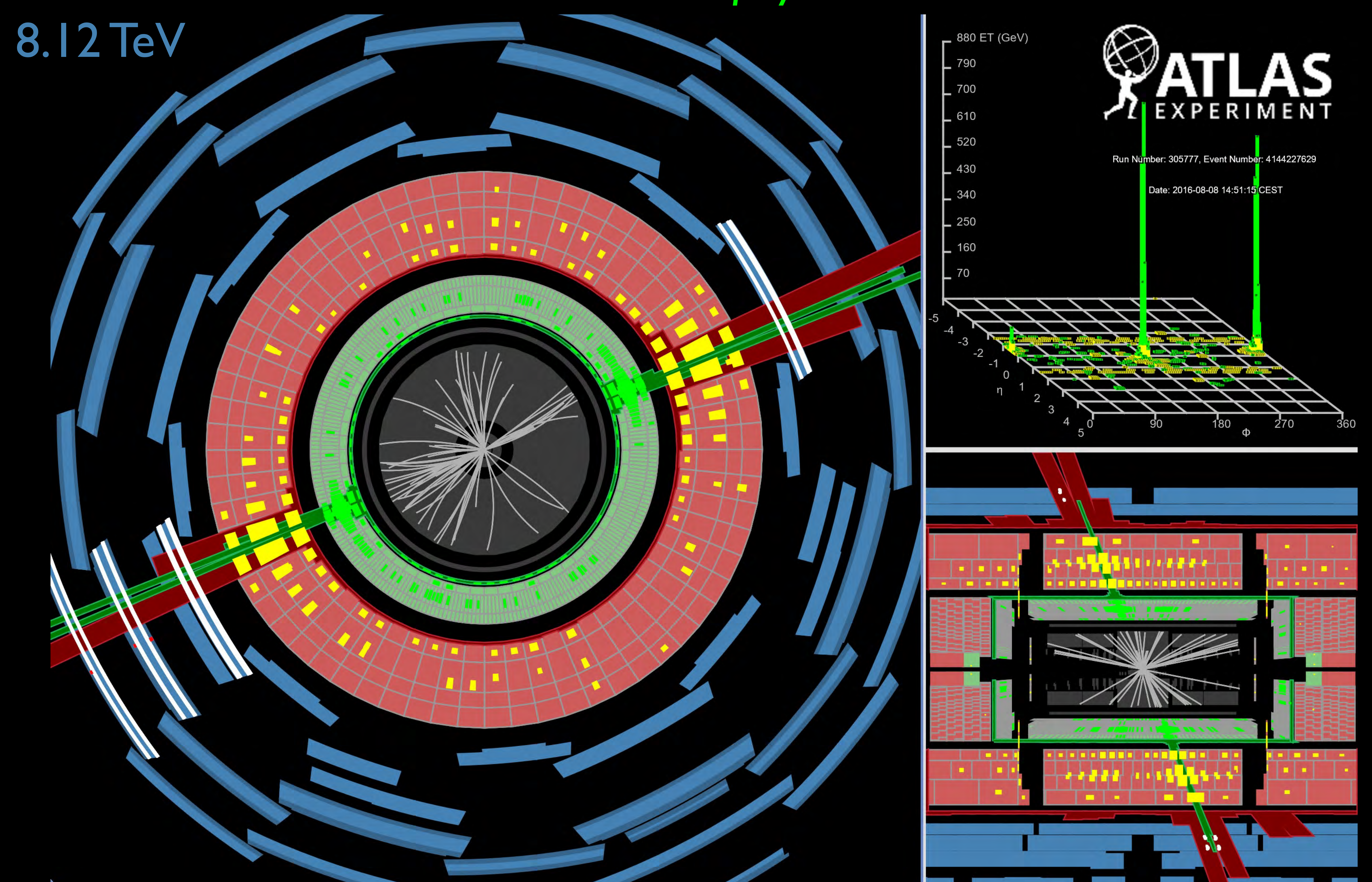




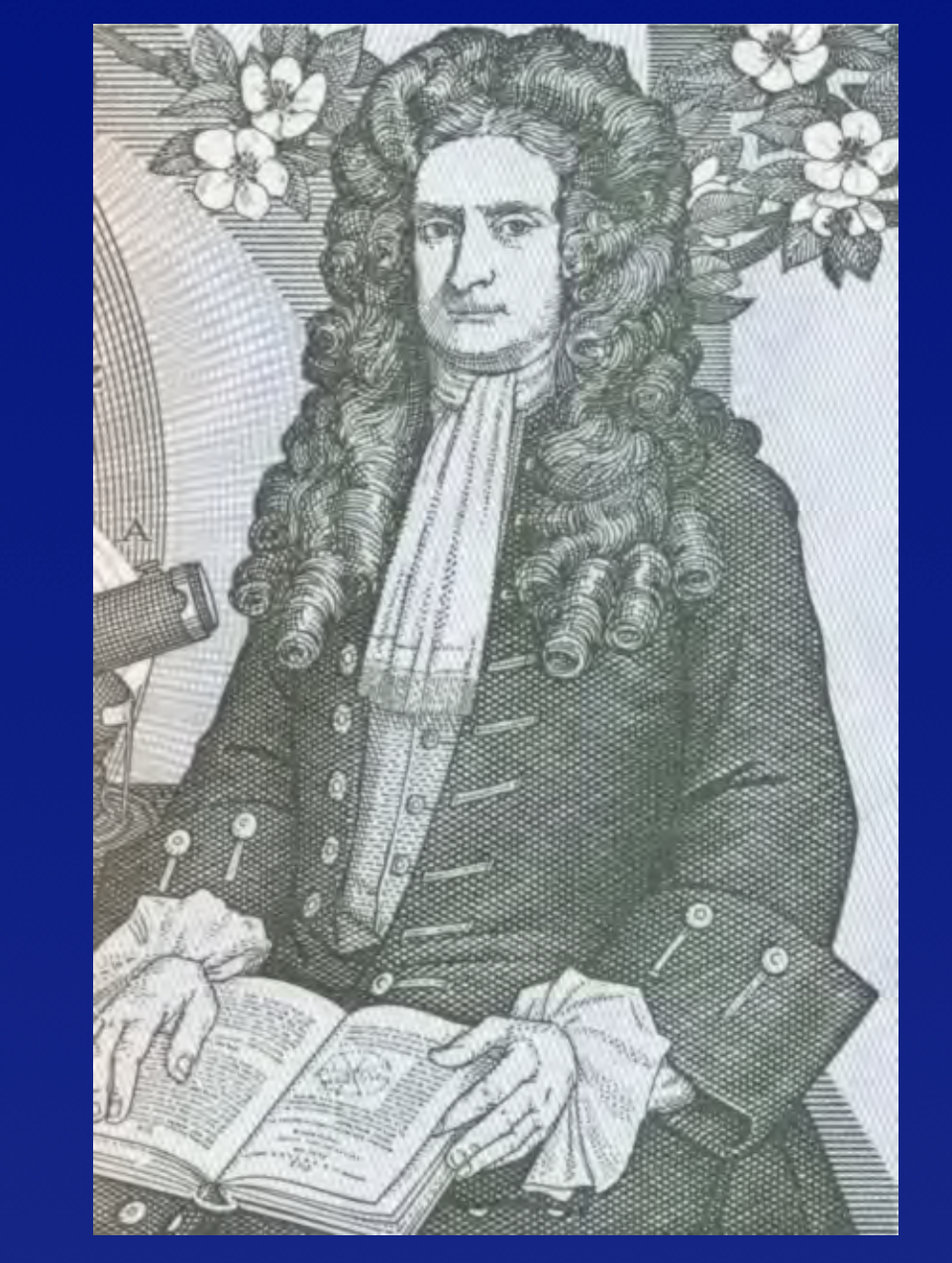

sum of parts

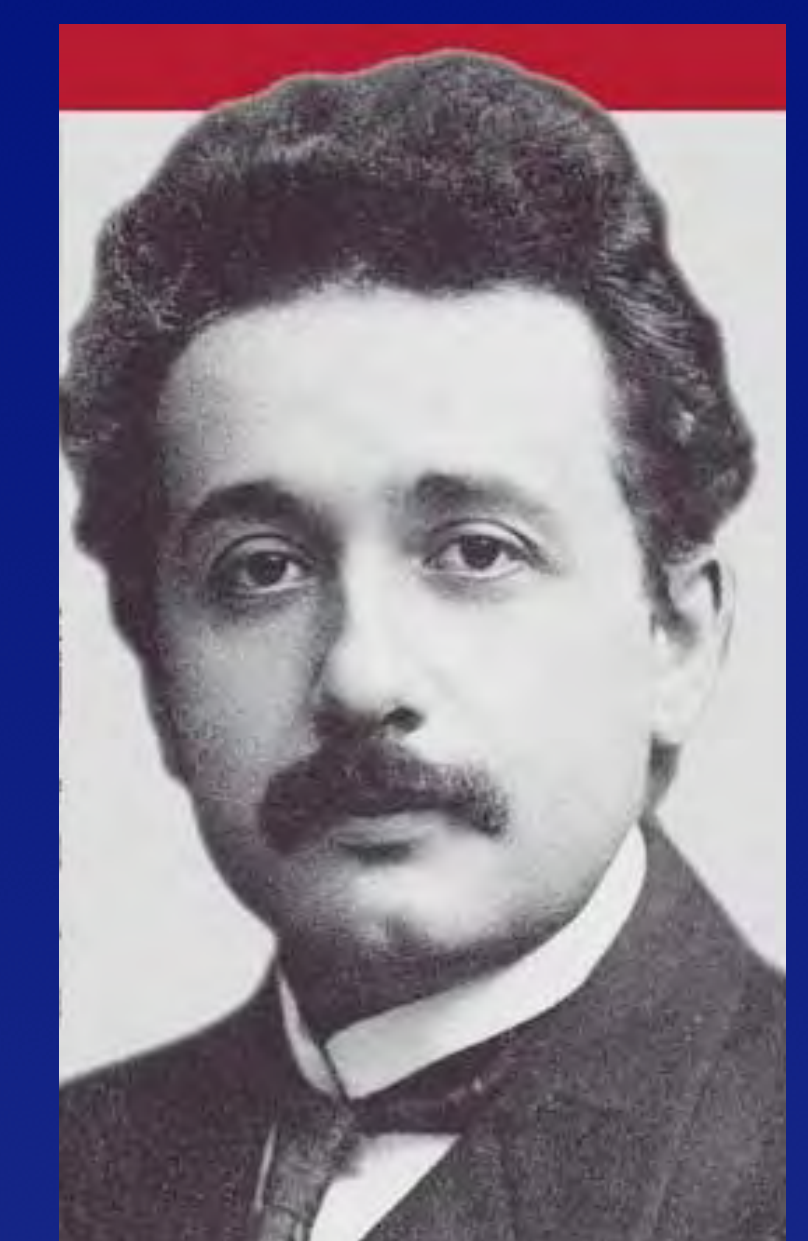

rest energy

Nucleon mass ( 940 MeV): exemplar of $m=E_{0} / c^{2}$ up and down quarks contribute few $\%$

$$
3 \frac{\mathrm{m}_{\mathrm{u}}+\mathrm{m}_{\mathrm{d}}}{2}=10 \pm 2 \mathrm{MeV}
$$

XPT: $M_{N} \longleftrightarrow 870 \mathrm{MeV}$ for massless quarks 
Lattice QCD: color-cỏnfinement origin of nucleon mass has explained nearly all visible mass in the Universe

(Quark masses 'ensure $M_{p}<M_{n}$ ) 
QCD could be complete*, up to M Planck

... but that doesn't prove it must be Prepare for surprises!

\section{How might QCD Crack?}

(Breakdown of factorization) Free quarks / unconfined color New kinds of colored matter Quark compositeness

Larger color symmetry containing QCD 


\section{New phenomena within QCD?}

Multiple production beyond diffraction + short-range order?

High density of few-GeV partons ... thermalization?

Long-range correlations in $y$ ?

Unusual event structures ...

Look at events in informative coordinates.

More is to be learned from the river of events than from a few specimens! 


\section{New spectroscopy of quarkonium-associated states}

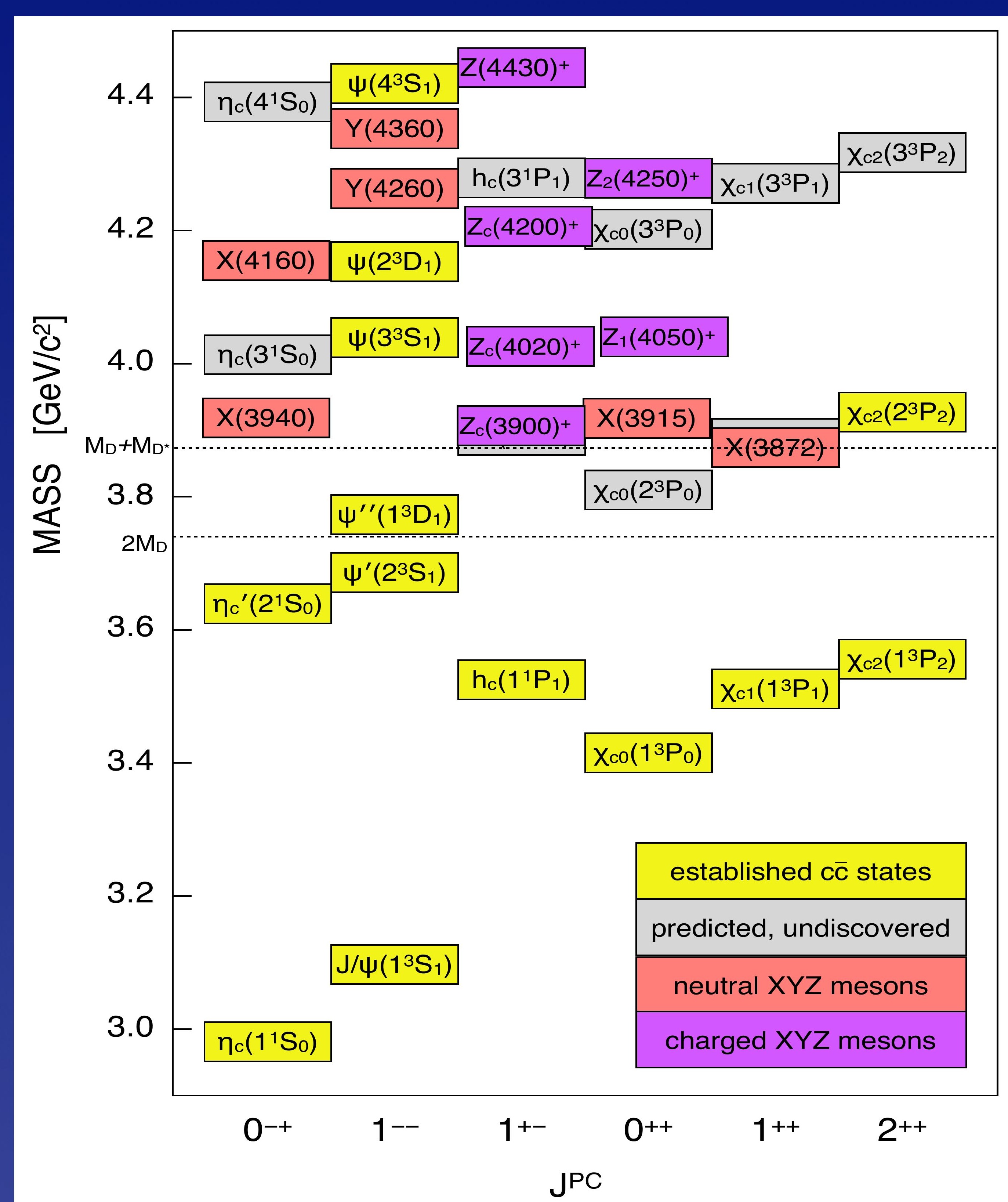

Stable doubly heavy tetraquark mesons

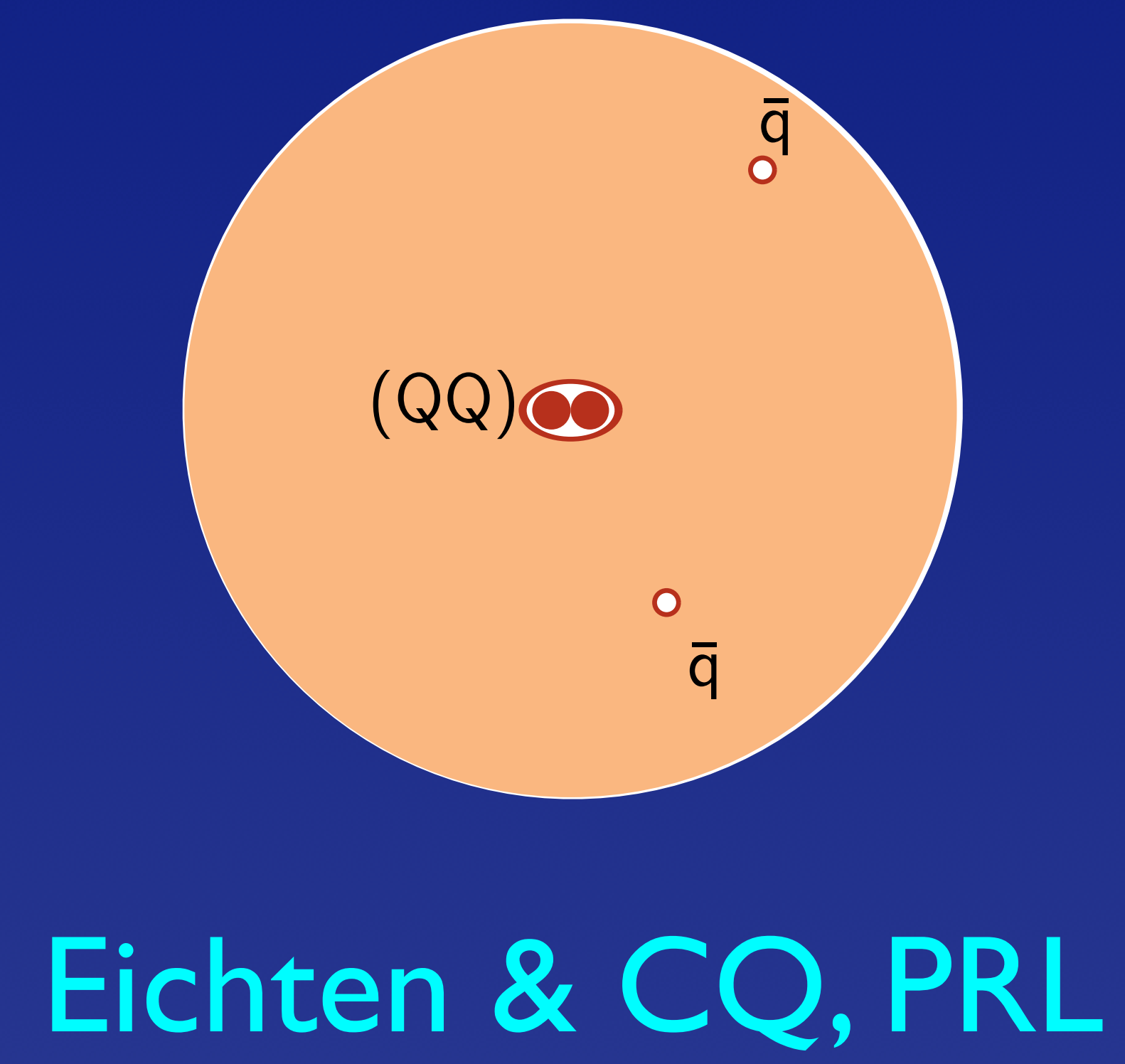




\section{Electroweak Symmetry Breaking}

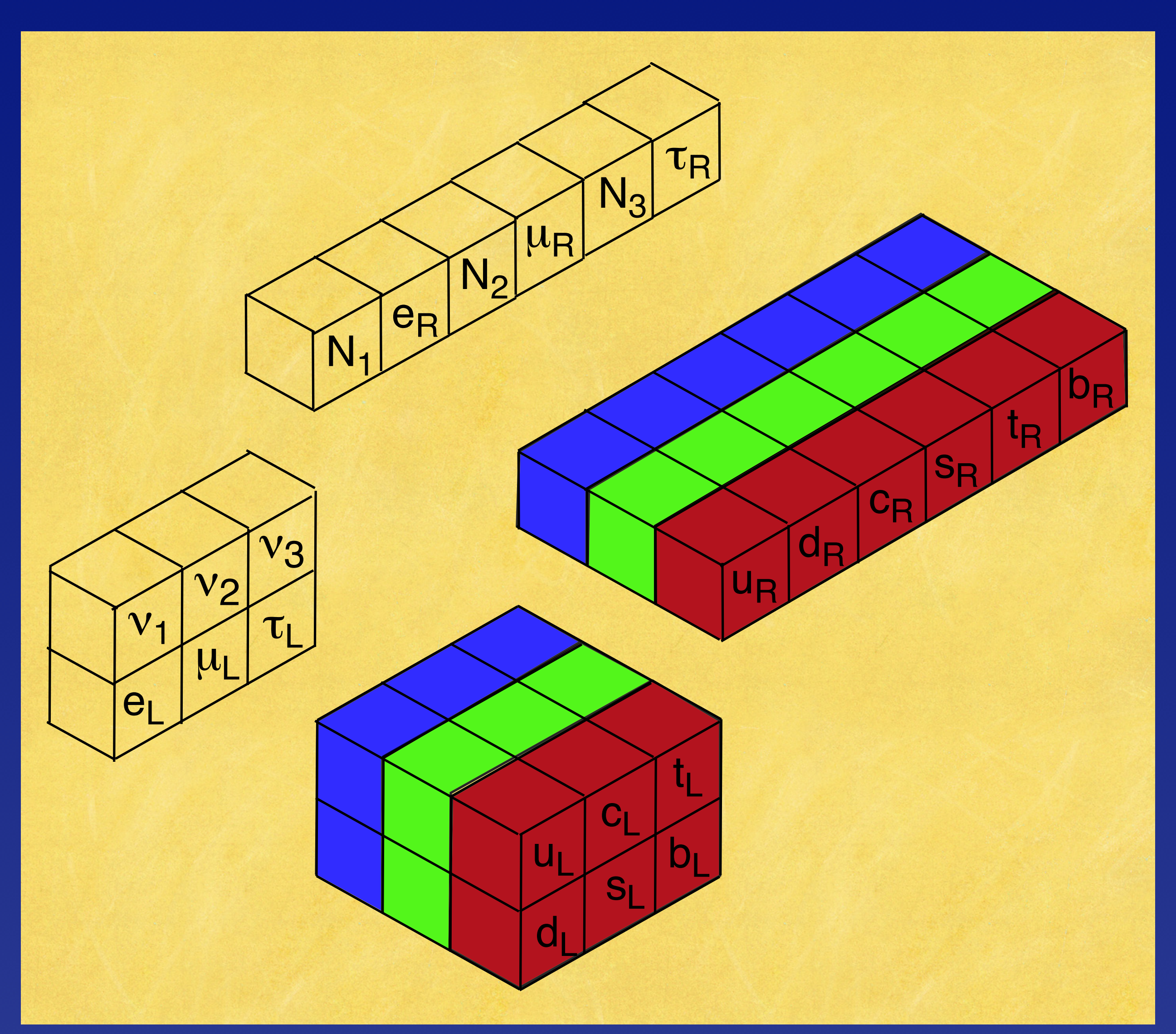

Interactions: $\mathrm{SU}(3)_{c} \otimes \mathrm{SU}_{(2)_{L} \otimes U} \otimes U(1)_{Y}$ gauge symmetries 


\section{The Importance of the I-TeV Scale}

EW theory does not predict Higgs-boson mass

Thought experiment: conditional upper bound

$$
\begin{gathered}
W^{+} W-, Z Z, H H, H Z \text { satisfy s-wave unitarity, } \\
\text { provided } M_{H} \leq\left(8 \pi \sqrt{2} / 3 G_{F}\right)^{1 / 2} \approx I T e V
\end{gathered}
$$

If bound is respected, perturbation theory is everywhere" reliable If not, weak interactions among $W^{ \pm}, Z, H$ become strong on I-TeV scale New phenomena are to be found around I TeV 


\section{Evolution of CMS 4-lepton Signal}

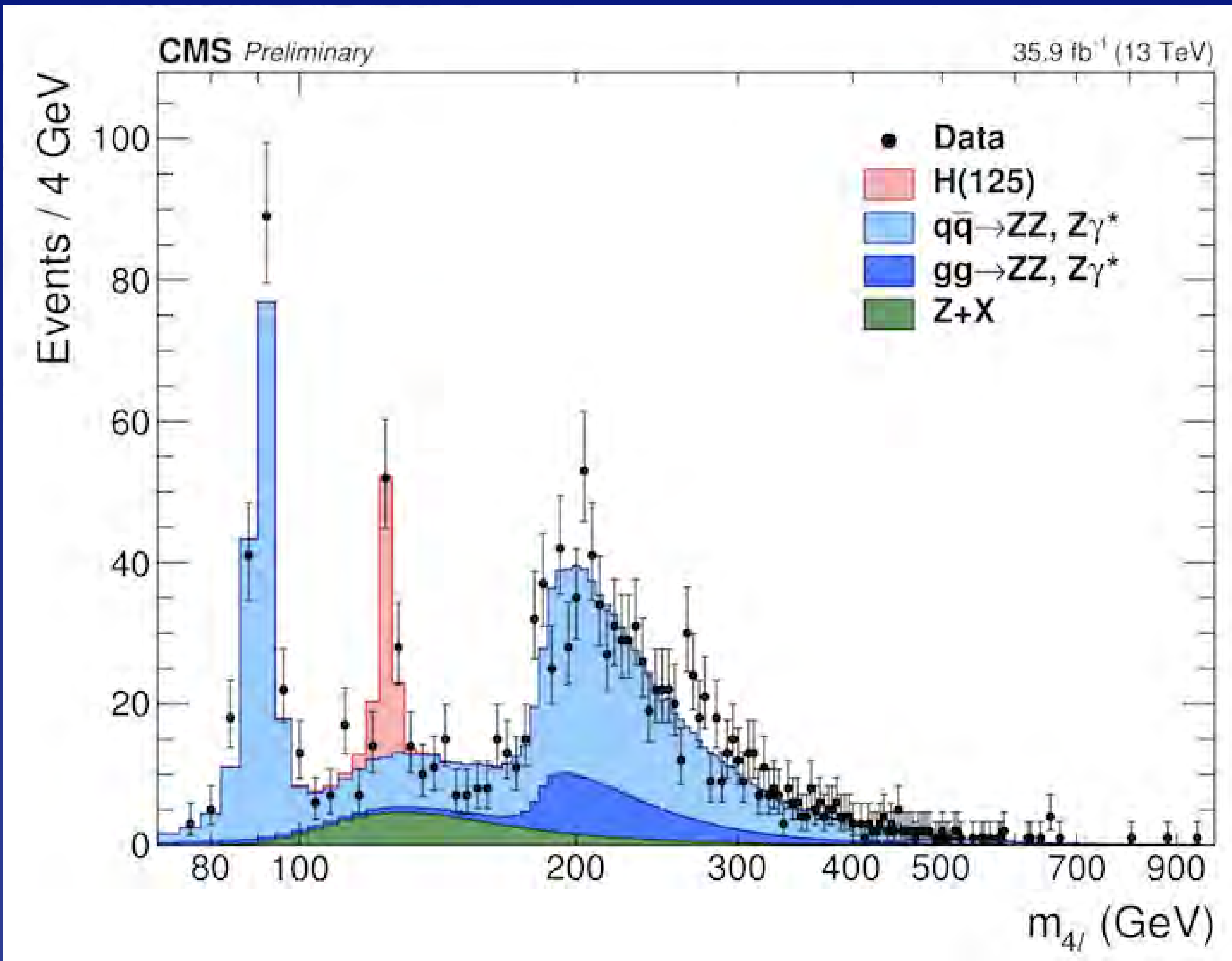




\section{LHC can study Higgs boson in many channels}
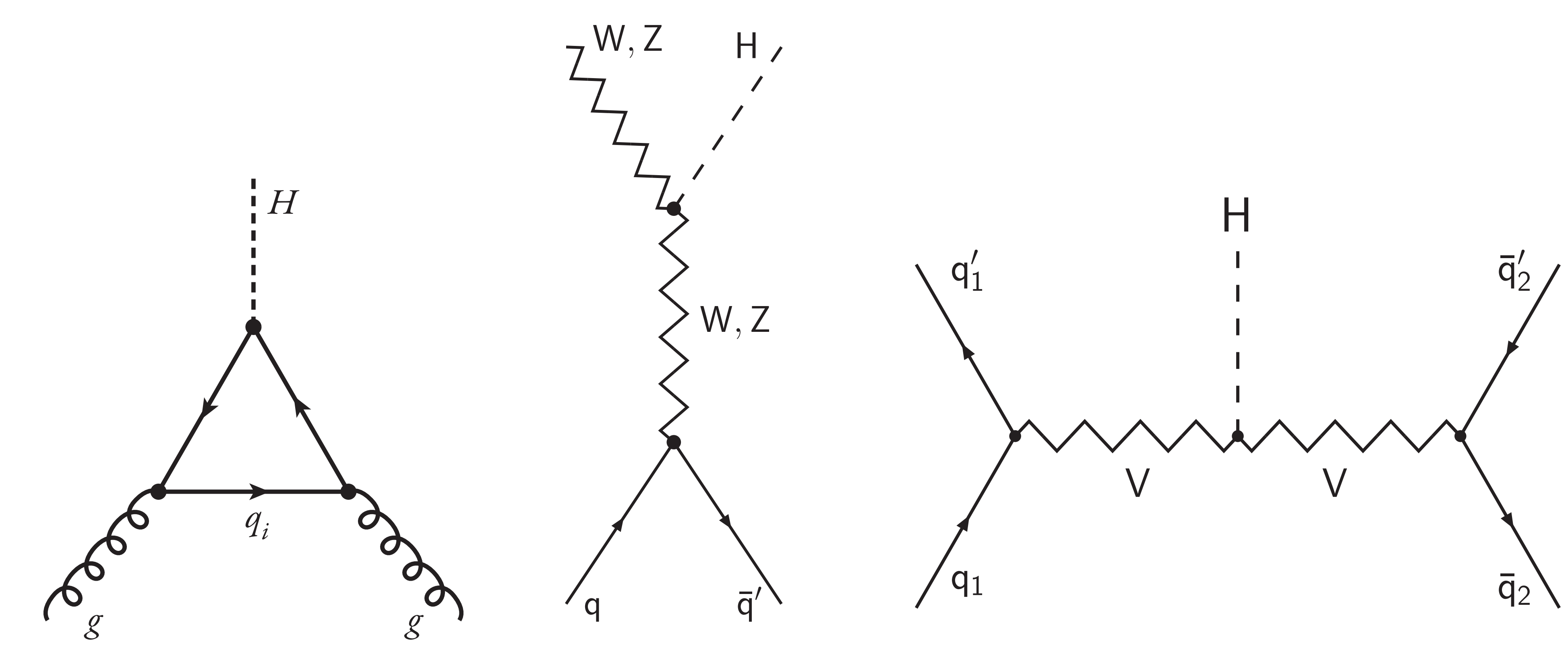

$+H t \bar{t}$

$\mathrm{V}, \mathrm{WW}^{*}, Z^{*}, \mathrm{~T}^{+} \mathrm{T}^{-}, b$ pairs,.. 


\section{Evolution of ATLAS Vy Signal}

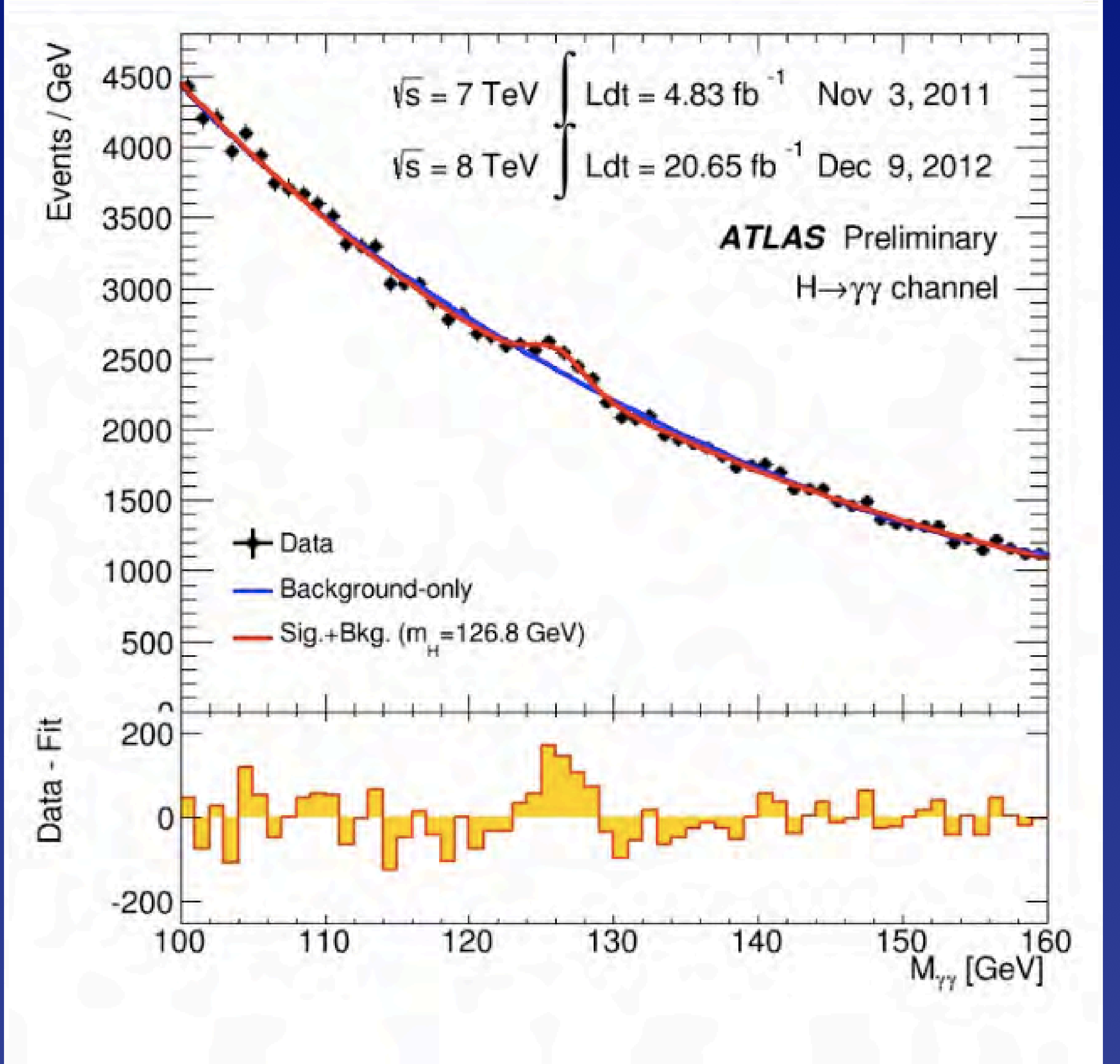




\section{What the LHC has told us about $H$ so far}

Evidence is developing as it would for a "standard-model" Higgs boson

\section{Unstable neutral particle near I25 GeV}

Motivates HL-LHen Higgs factory
electron-positron ded

$$
M_{H}=125.09 \pm 0.24 \mathrm{GeV}
$$

$$
\begin{aligned}
& \text { decays to } \mathrm{YV}, \mathrm{W}^{+} \mathrm{W}^{-}, \mathrm{ZZ} \\
& \text { dominantly spin-parity } 0^{+}
\end{aligned}
$$

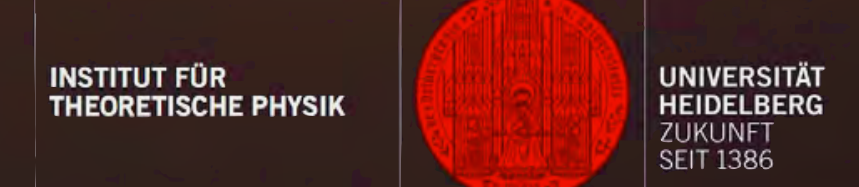

Nov6-10 2017 HIGGS COUPLINGS

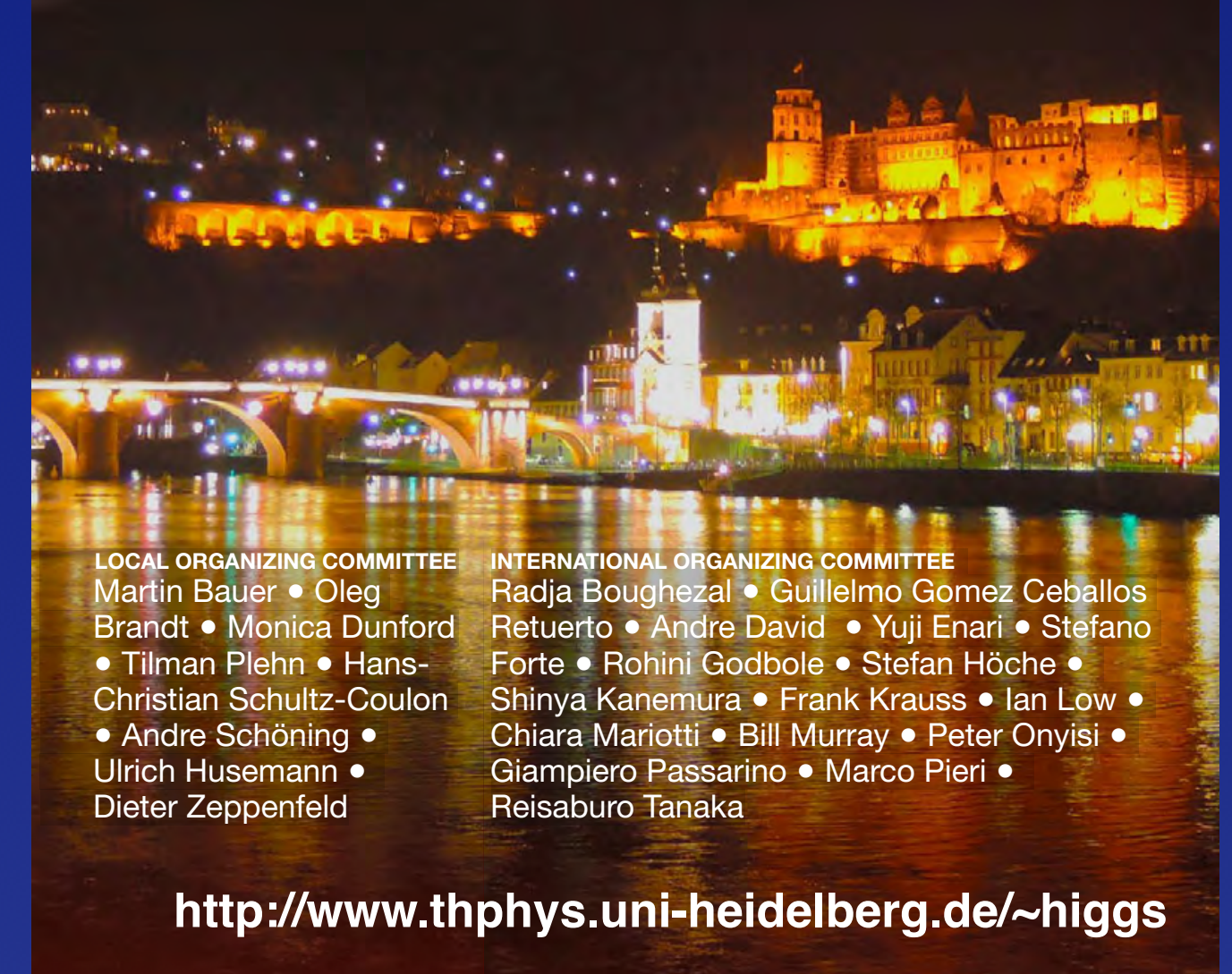

evidence for $T^{+} T^{-}, b \bar{b}, t \bar{t} ; \mu^{+} \mu^{-}$limited

Only third-generation fermions tested 
Quantum corrections test electroweak theory

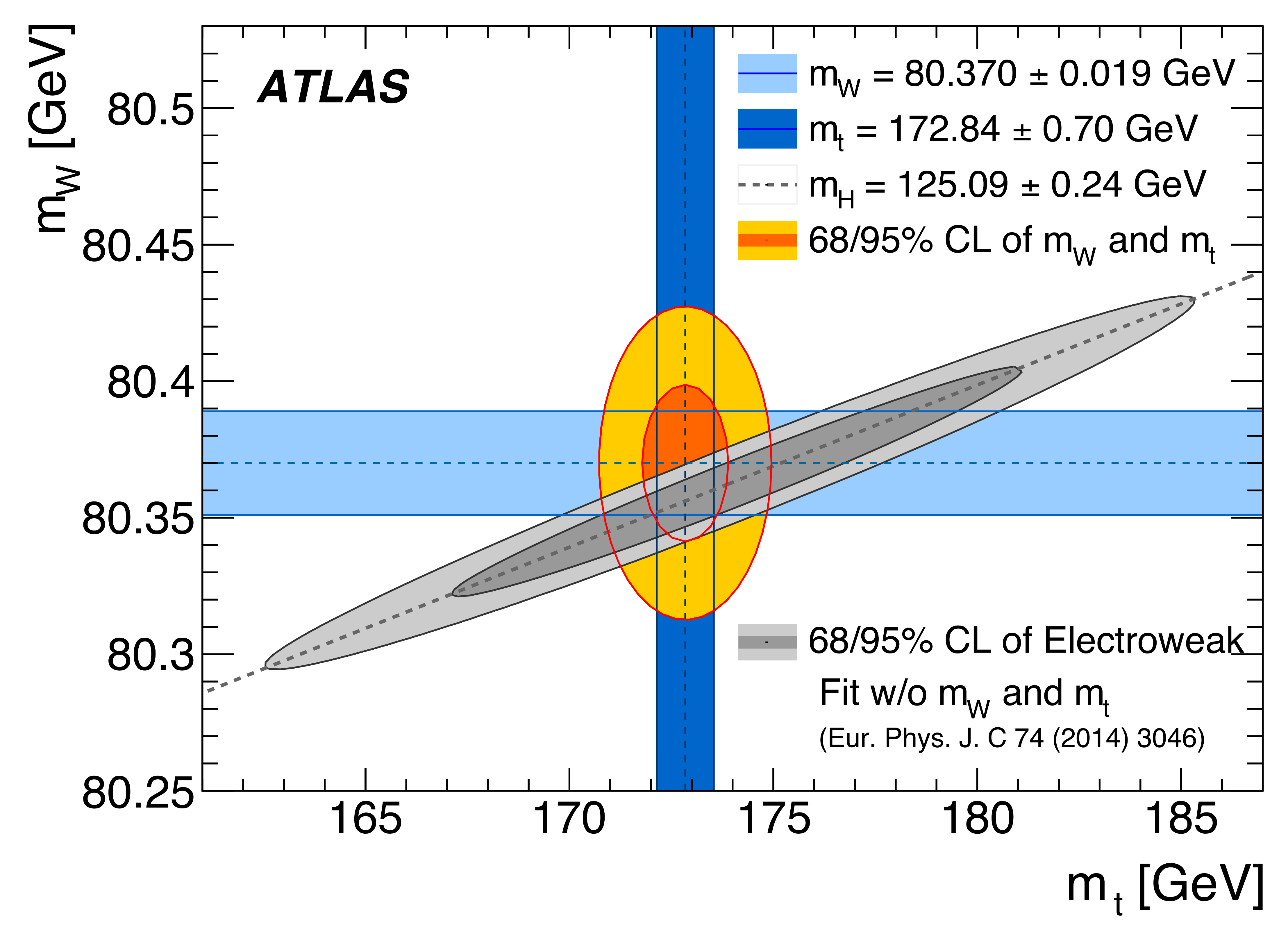


Why does discovering the agent matter?

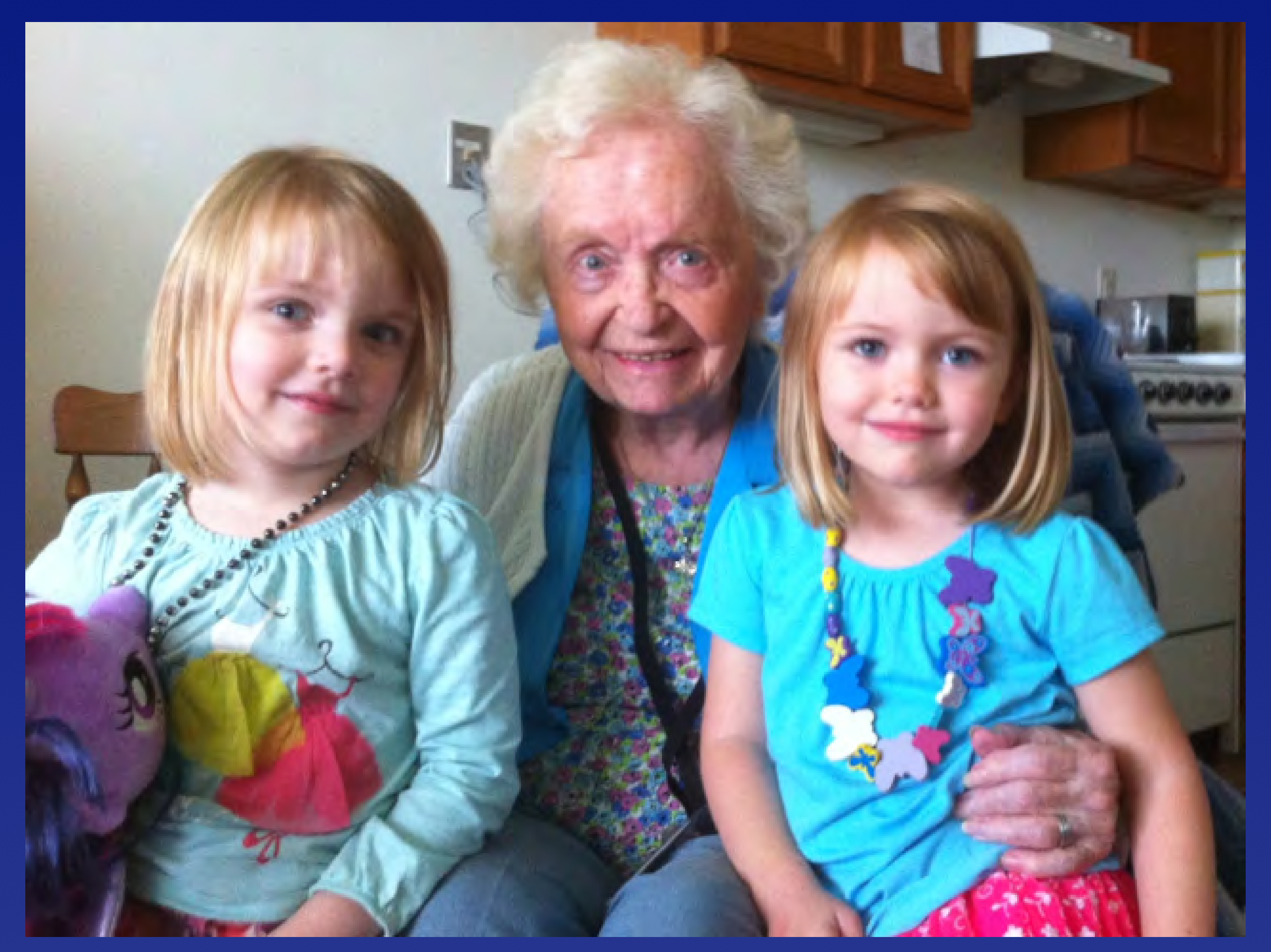

Imagine a world without a symmetry-breaking (Higgs) mechanism at the electroweak scale 
Electron and quarks would have no mass via Higgs

QCD would confine quarks into protons, etc.

Nucleon mass little changed

Surprise: QCD would hide EW symmetry,

give tiny masses to $W, Z$

Massless electron: atoms lose integrity

No atoms means no chemistry, no stable

composite structures like liquids, solids, ...

... no template for life. 


\section{What we expect of the standard-model Higgs sector}

Hide electroweak symmetry

Give masses to W, Z, H

Regulate Higgs-Goldstone scattering

$\left.\begin{array}{l}\text { Account for quark masses, mixings } \\ \text { Account for charged-lepton masses }\end{array}\right\}$ ФBSM

A role in neutrino masses? 
Fully accounts for EWSB (W, Z couplings)?

Couples to fermions?

$t$ from production, $H t \bar{t}$

need direct observation for $b, T$

Accounts for fermion masses?

Fermion couplings $\propto$ masses?

Are there others?

Quantum numbers? $\left(\mathrm{J}^{\mathrm{P}}=0^{+}\right)$

SM branching fractions to gauge bosons?

Decays to new particles?

All production modes as expected?

Implications of $M_{H} \approx 125 \mathrm{GeV}$ ?

Any sign of new strong dynamics? 
More new physics on the TeV scale? WIMP dark matter "Naturalness"

Hierarchy problem: EW scale « Planck scale Vacuum energy problem Clues to origin of EWSB 


\section{Direct searches for WIMP dark matter}

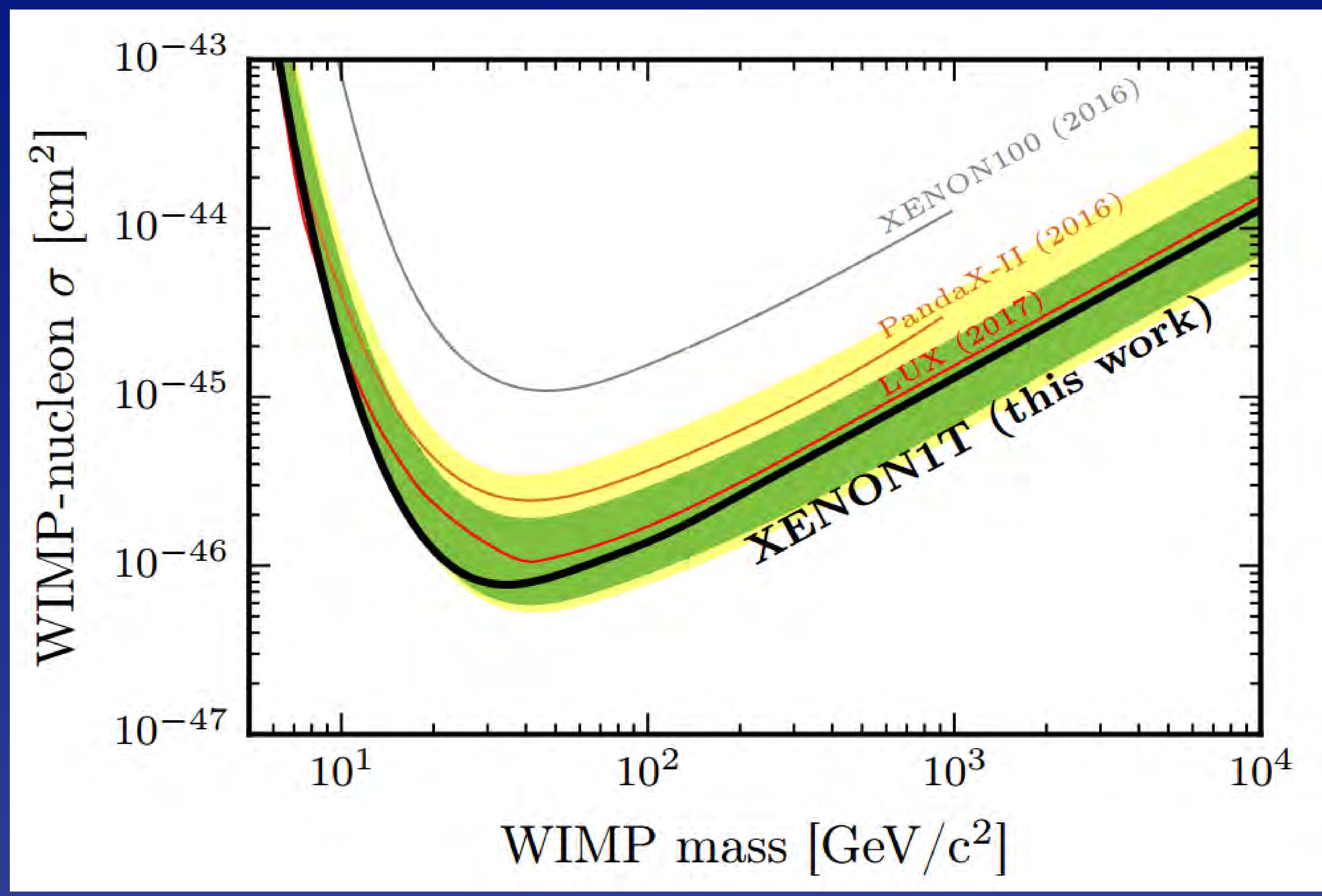




\title{
Supersymmetry could respond to many SM problems, but (as we currently understand it) it is largely unprincipled!
}

\author{
$R$-parity (overkill for proton stability) \\ gives dark-matter candidate \\ $\mu$ problem (getting $\mathrm{Te} V$ scale right) \\ Taming flavor-changing neutral currents \\ All these are added by hand!
}

Very promising: search in EW production modes reexamine squark + EWino, too. 


\section{How have we misunderstood the hierarchy problem?}

If other physical scales are present, there is something to understand

We originally sought once-and-done remedies, such as supersymmetry or technicolor

Go in steps, or reframe the problem? 


\section{The unreasonable effectiveness of the standard model}




\section{A Unified Theory?}

Why are atoms so remarkably neutral?

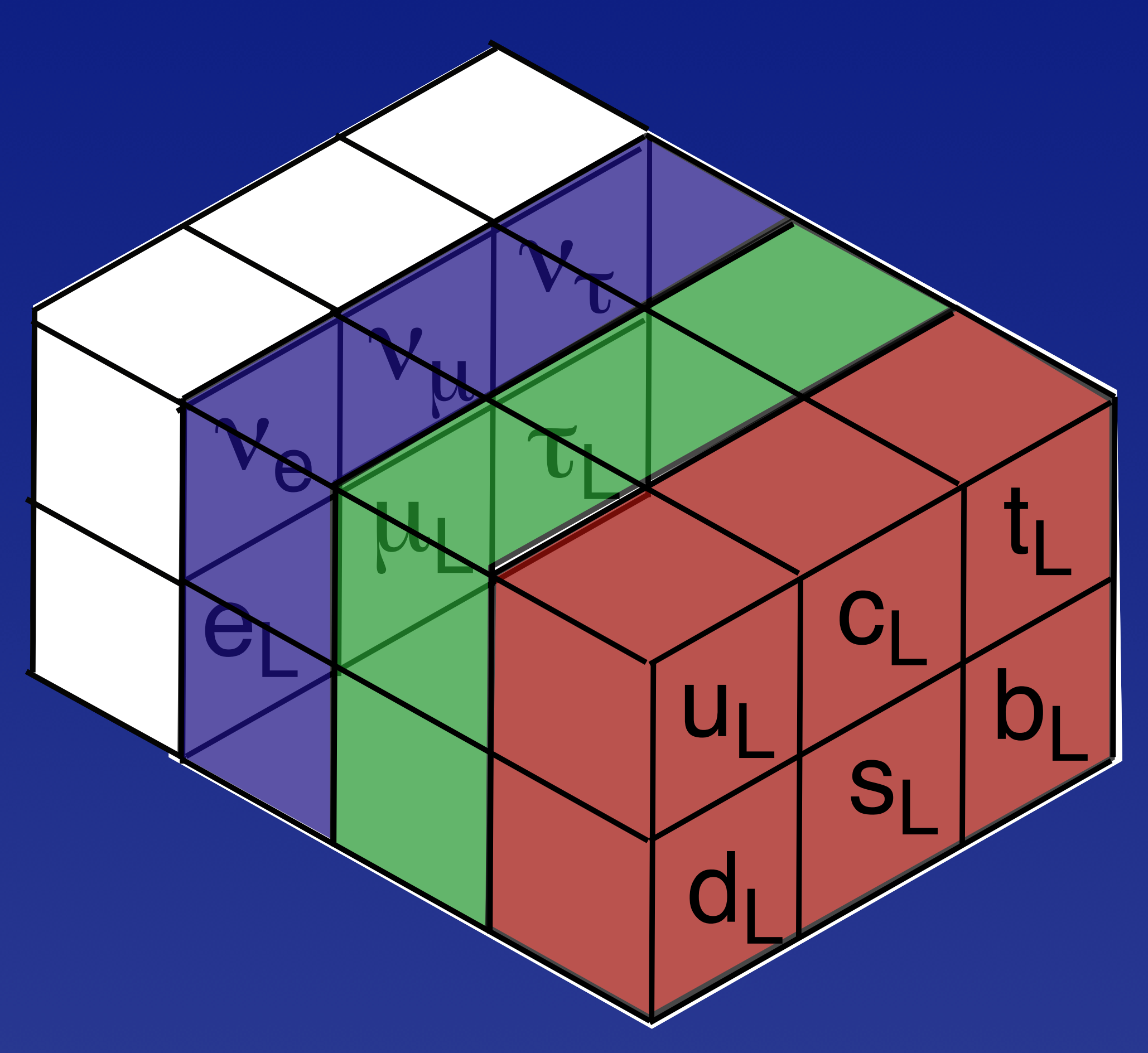

Coupling constant unification?

Extended quark-lepton families: proton decay! $n-\bar{n}$ oscillations 
Unification of Forces?

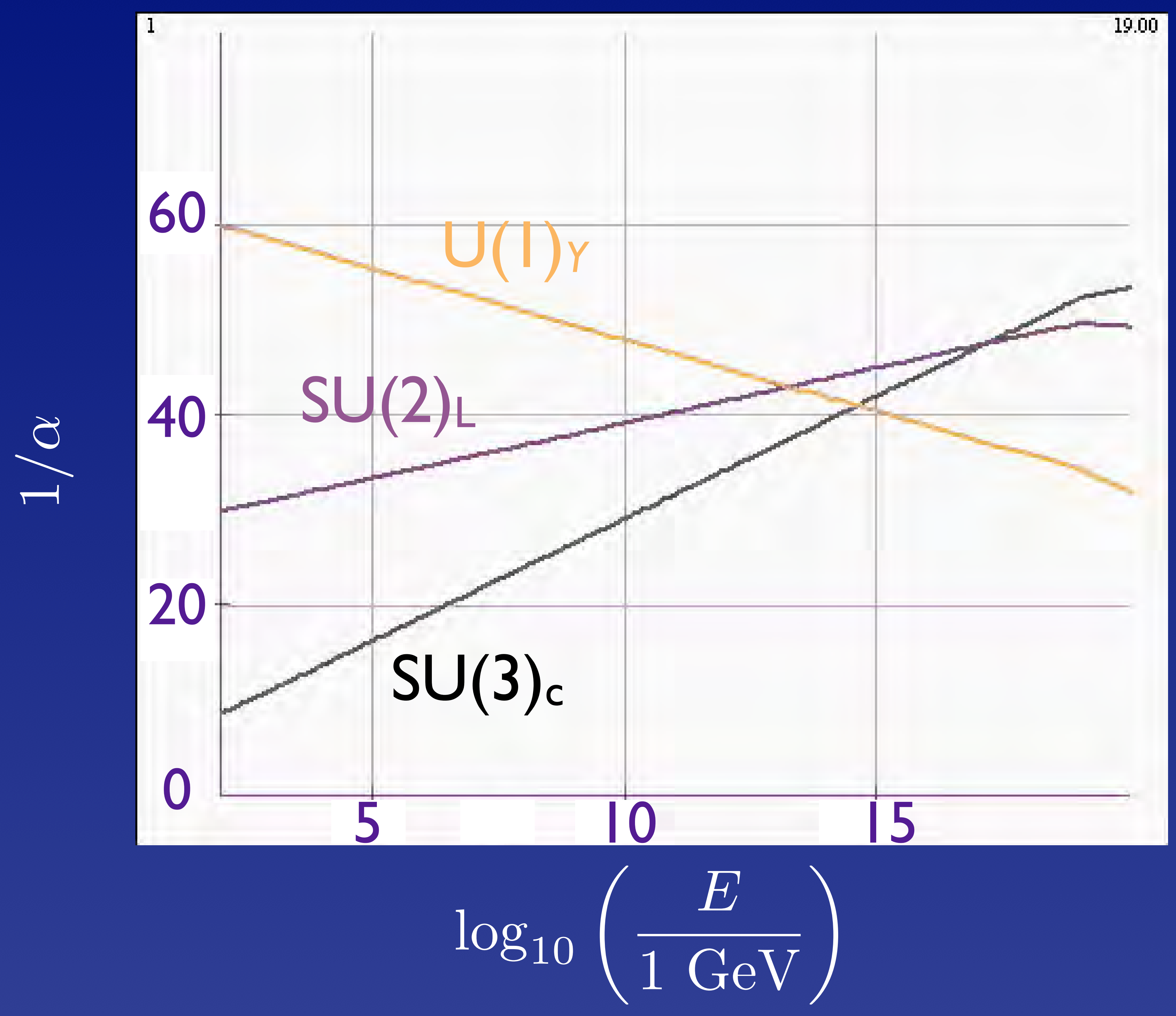


Might (HE-)LHC (or $100-\mathrm{TeV}$ ) see change in evolution?

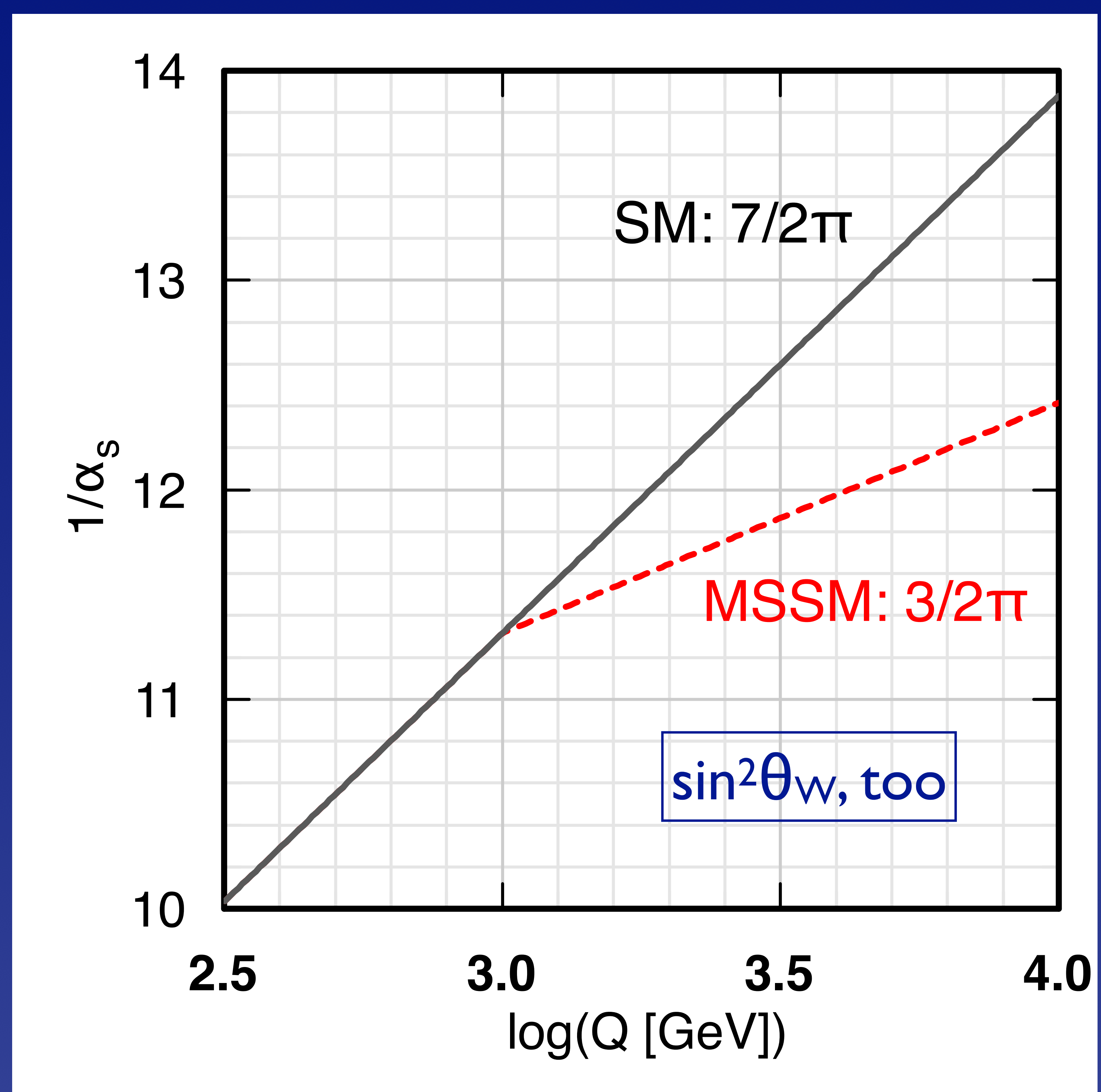




\section{Parameters of the Standard Model}

3 coupling parameters $\alpha_{s}, \alpha_{\mathrm{em}}, \sin ^{2} \theta_{W}$

2 parameters of the Higgs potential

1 vacuum phase (QCD)

6 quark masses

3 quark mixing angles

$1 \quad$ CP-violating phase

3 charged-lepton masses

3 neutrino masses

3 leptonic mixing angles leptonic CP-violating phase (+ Majorana ...)

$26^{+}$arbitrary parameters 
Why does the muon weigh?

$$
\text { gauge symmetry allows }
$$

$$
\begin{array}{r}
\zeta_{\mathrm{e}}\left[\left(\overline{\mathrm{e}}_{\mathrm{L}} \Phi\right) \mathrm{e}_{\mathrm{R}}+\overline{\mathrm{e}}_{\mathrm{R}}\left(\Phi^{\dagger} \mathrm{e}_{\mathrm{L}}\right)\right] \rightsquigarrow \mathrm{m}_{\mathrm{e}}=\zeta_{\mathrm{e}} \mathrm{v} / \sqrt{2} \\
\text { after spontaneous symmetry breaking }
\end{array}
$$

What does the muon weigh?

$\zeta_{e}:$ picked to give right mass, not predicted

fermion mass implies physics beyond the standard model 
Charged Fermion Masses

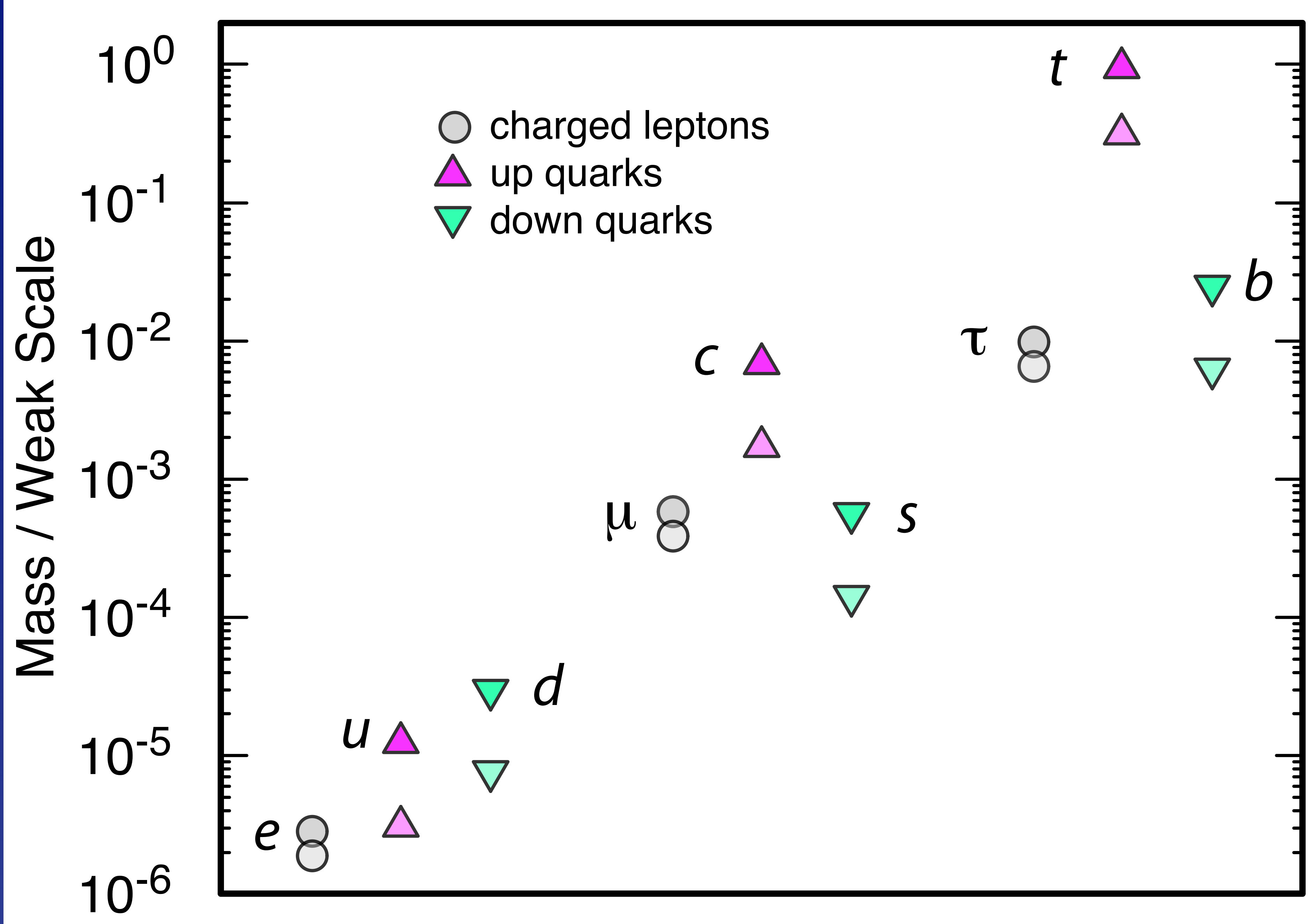

Running mass $m(m) \ldots m(U)$ 


\section{Quark family patterns: generations}

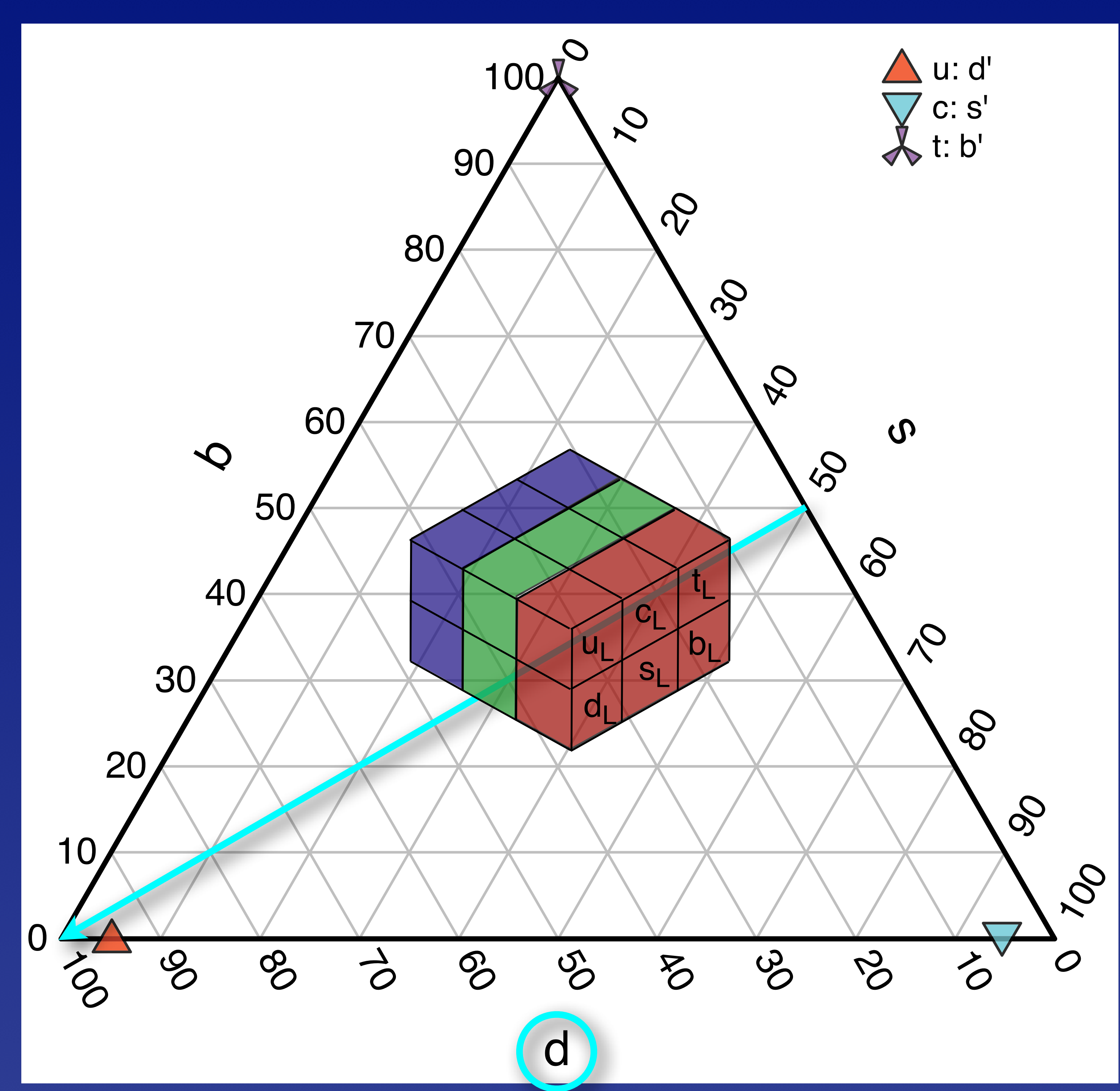


Rare Processes: Flavor-changing neutral currents

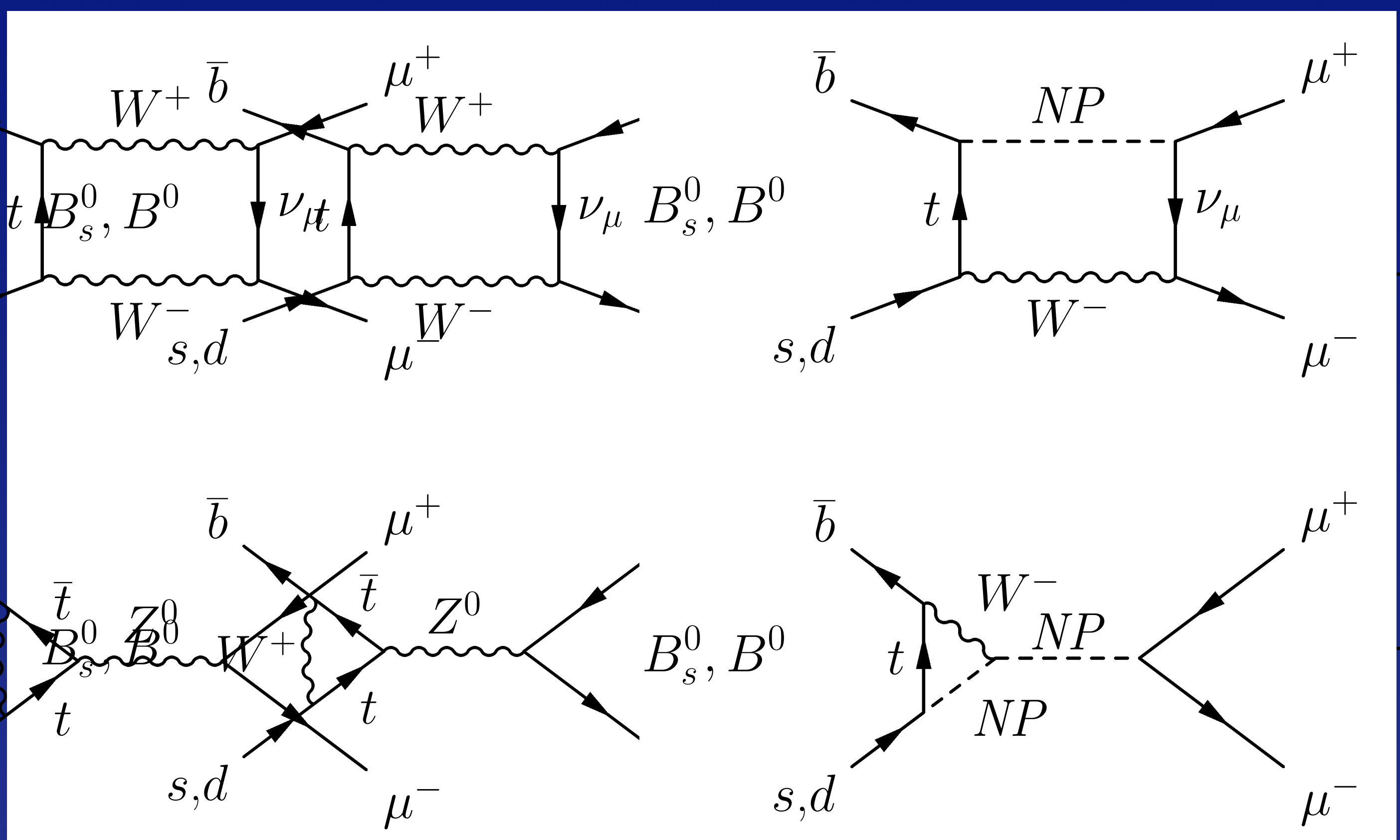

$\mathrm{SM}: \mathrm{BR}\left(\mathrm{B}_{\mathrm{s}} \rightarrow \mu^{+} \mu^{-}\right)=(3.65 \pm 0.30) \times 10^{-9}$

MSSM: $\operatorname{BR}\left(B_{s} \rightarrow \mu^{+} \mu^{-}\right) \propto \frac{m_{b}^{2} m_{t}^{2}}{M_{A}^{4}} \tan ^{6} \beta$ 


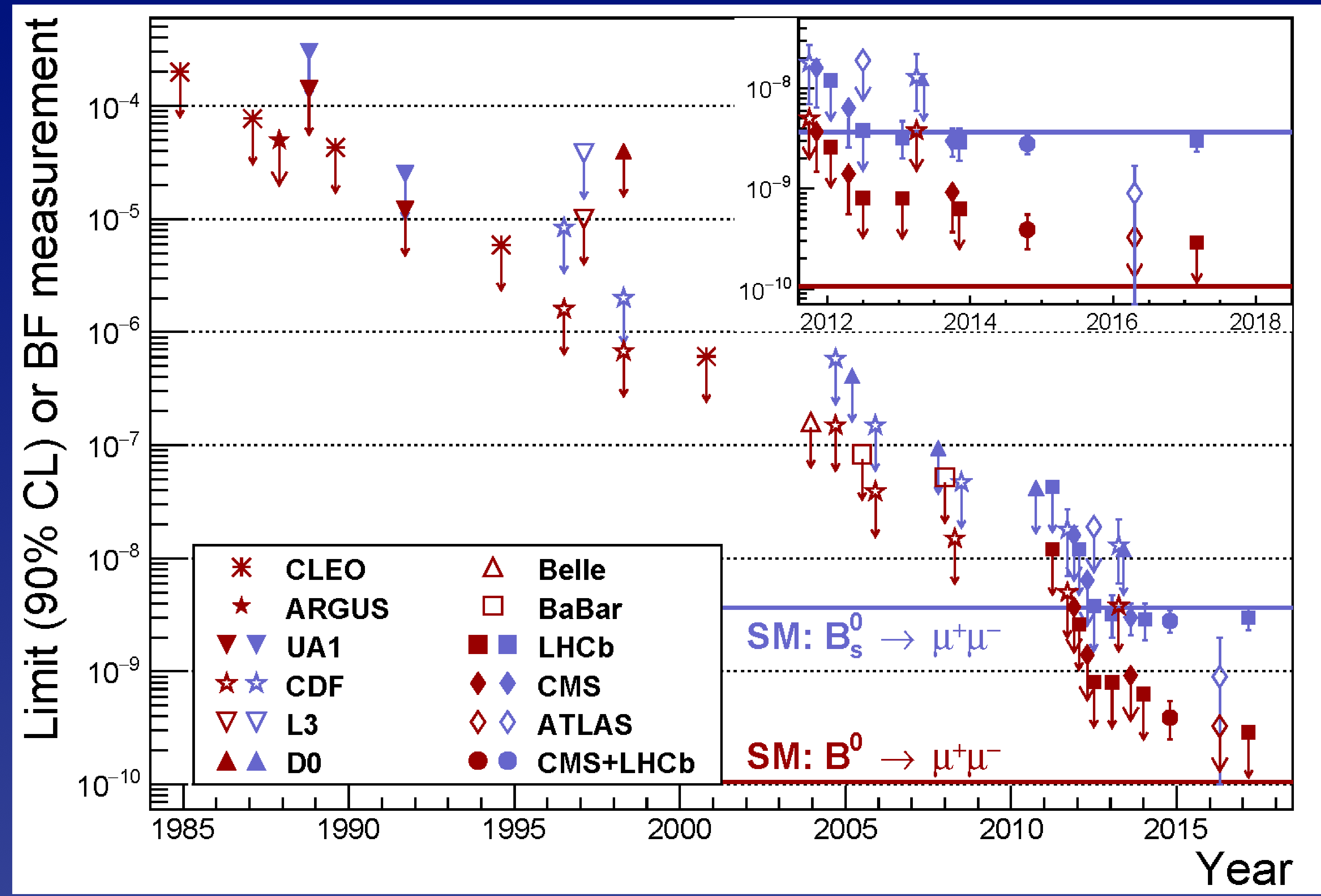


$\left(\mathrm{B}^{0}, \mathrm{~B}_{\mathrm{s}}\right) \rightarrow \mu^{+} \mu^{-}$

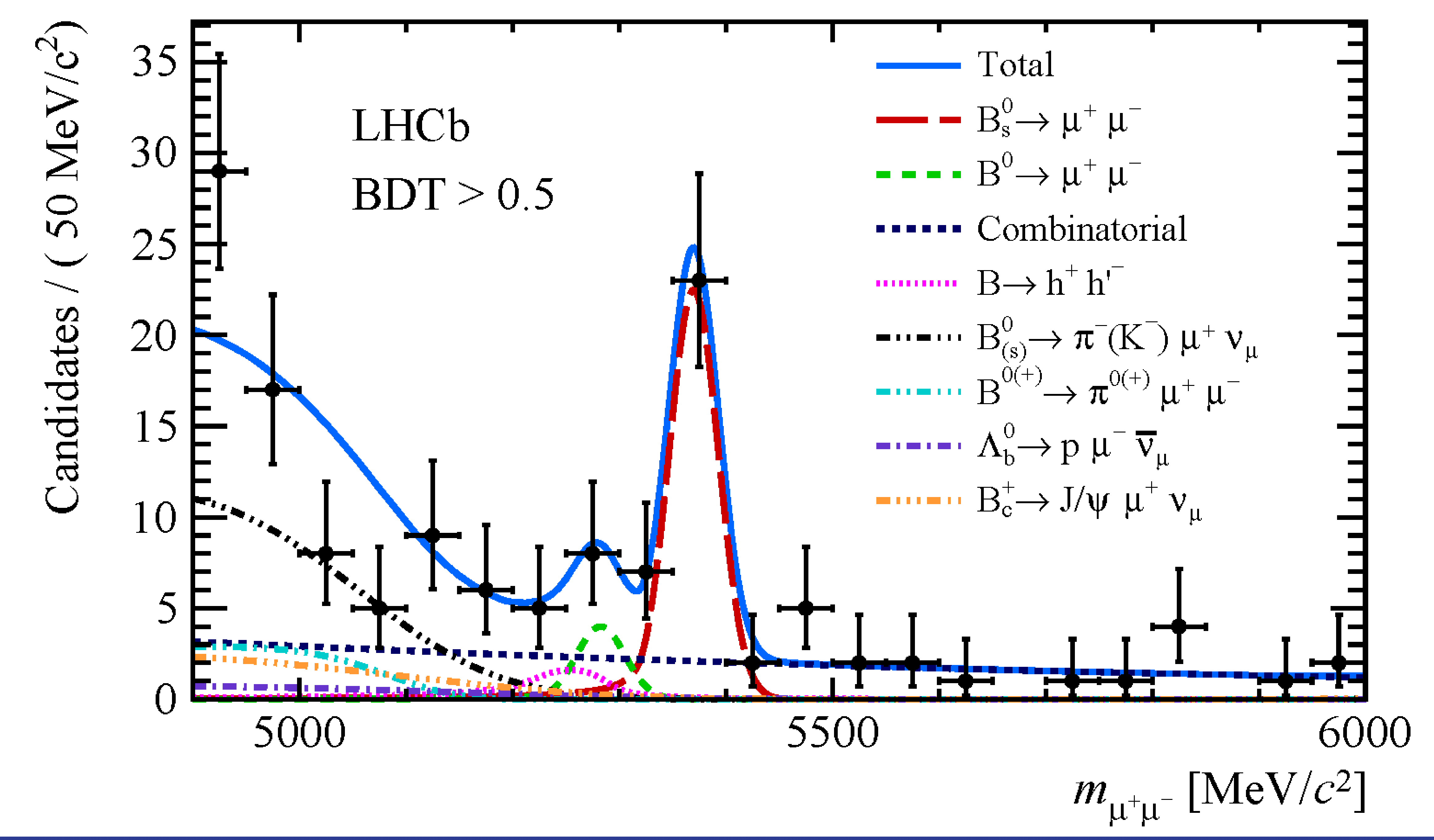

$\mathrm{LHCb}: \mathrm{BR}\left(\mathrm{B}_{\mathrm{s}} \rightarrow \mu^{+} \mu^{-}\right)=\left(3.0_{-0.6}^{+0.7}\right) \times 10^{-9}$ 


\section{Flavor anomalies}

\section{LHCb sees several hints of flavor nonuniversality}

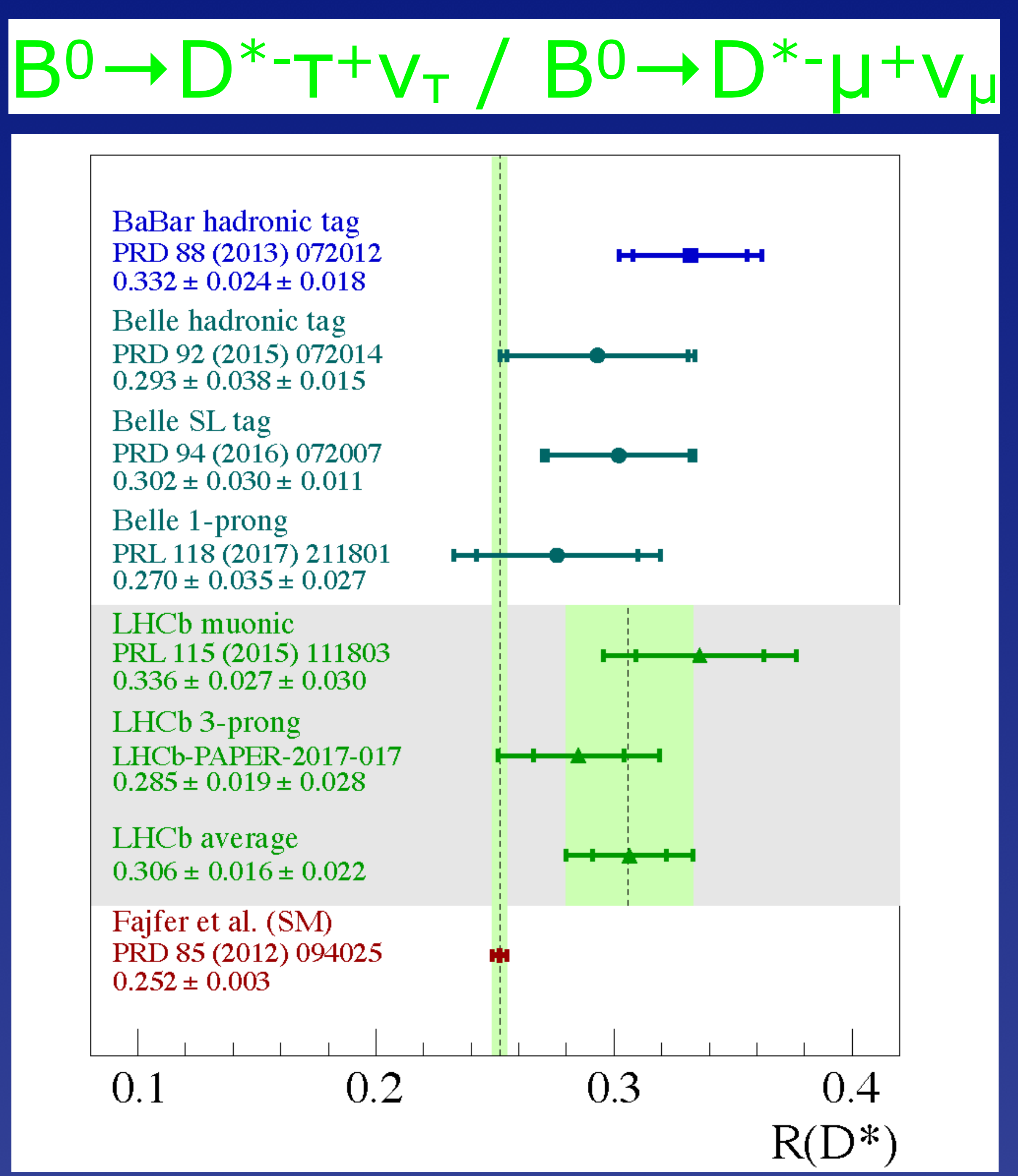

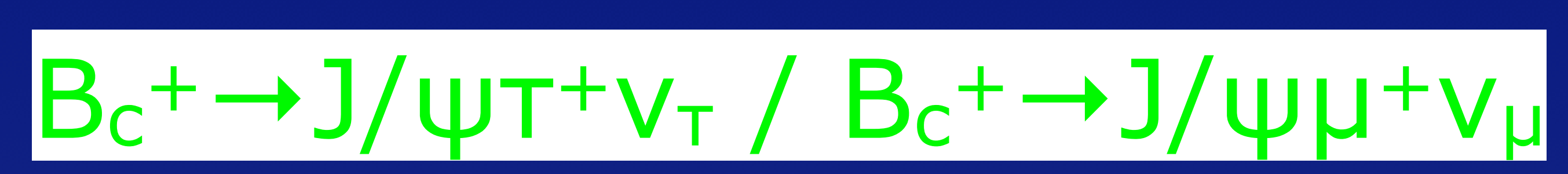

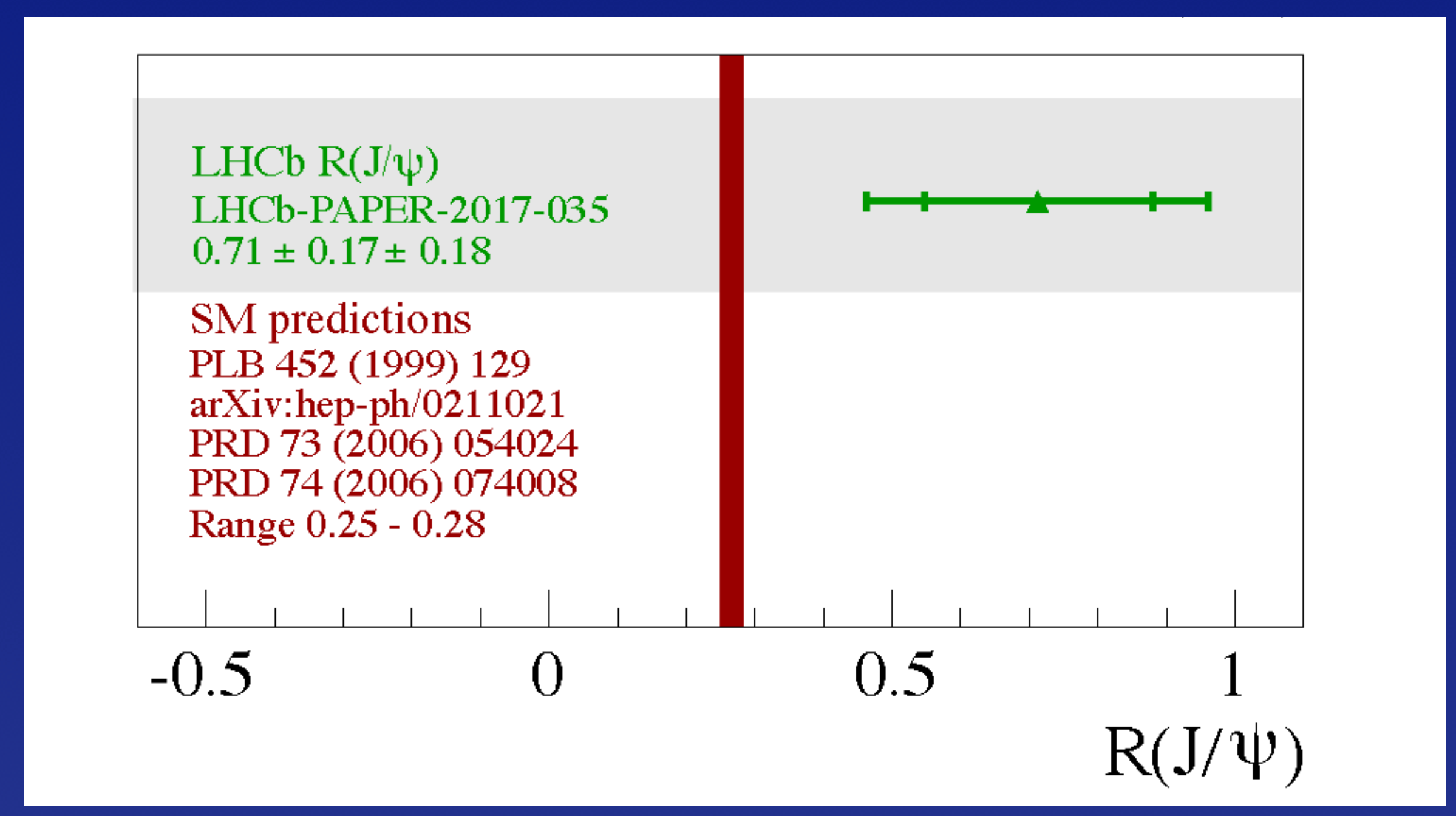

Too many $\mathrm{T}$; other evidence for excess $\mu^{+} \mu^{-} / \mathrm{e}^{+} \mathrm{e}^{-}$ 
Some outstanding questions in $\mathrm{V}$ physics

What is the composition of $V_{3}$ ?

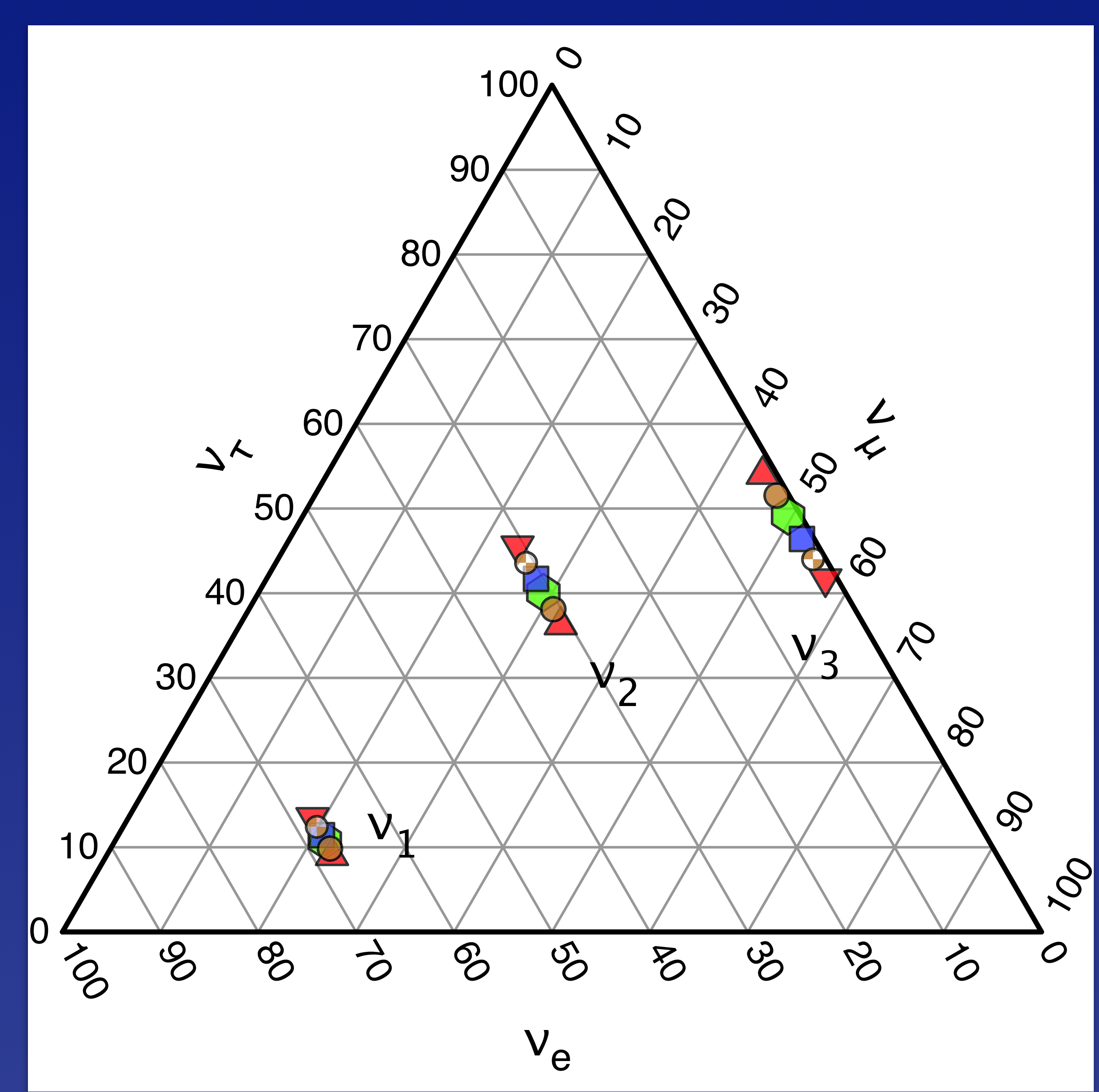

Before most-recent experiments 
Some outstanding questions in $\mathrm{V}$ physics What is the composition of $V_{3}$ ?

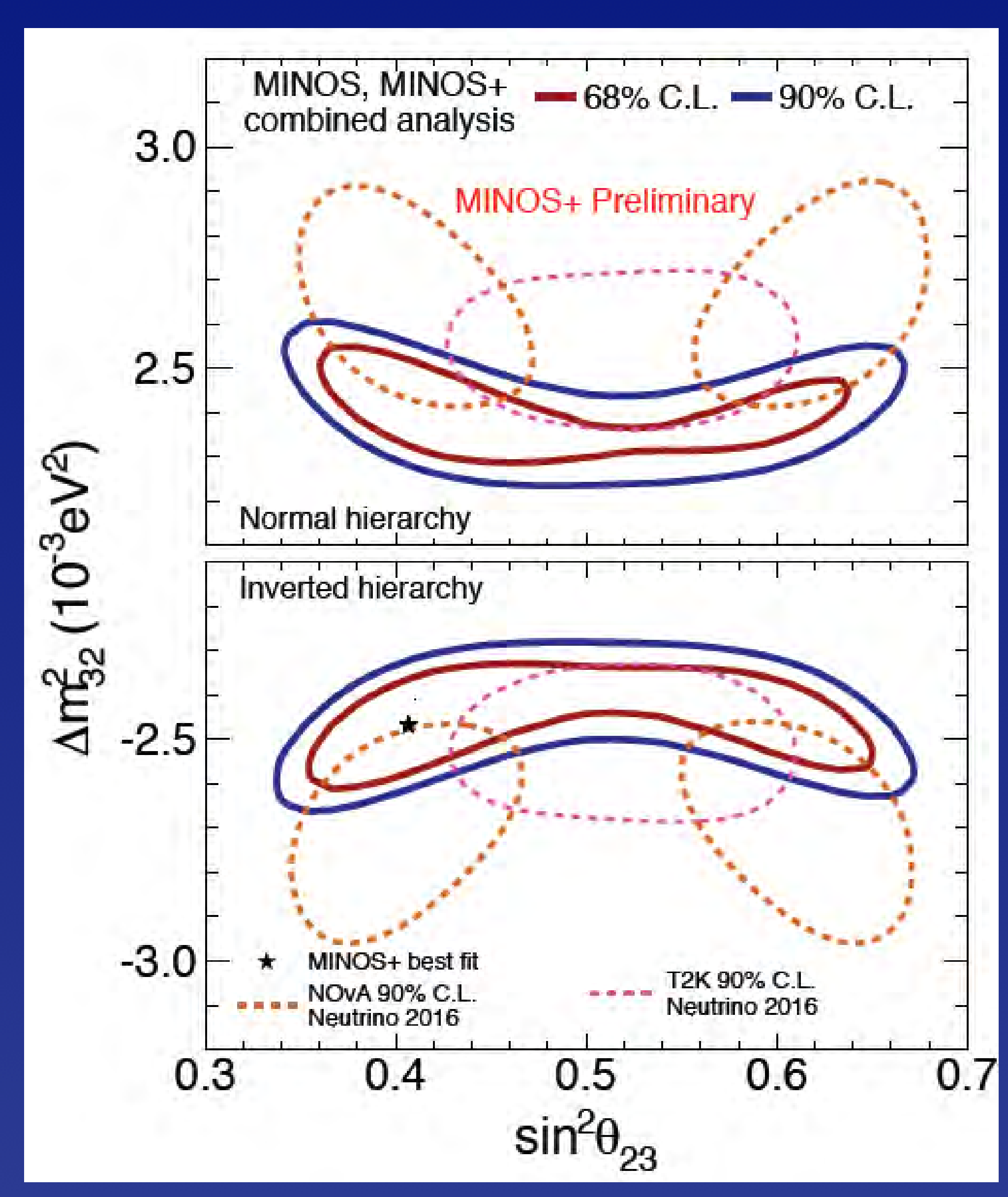

T2K favors maximal mixing, NOVA nonmaximal 


\section{Some outstanding questions in V physics}

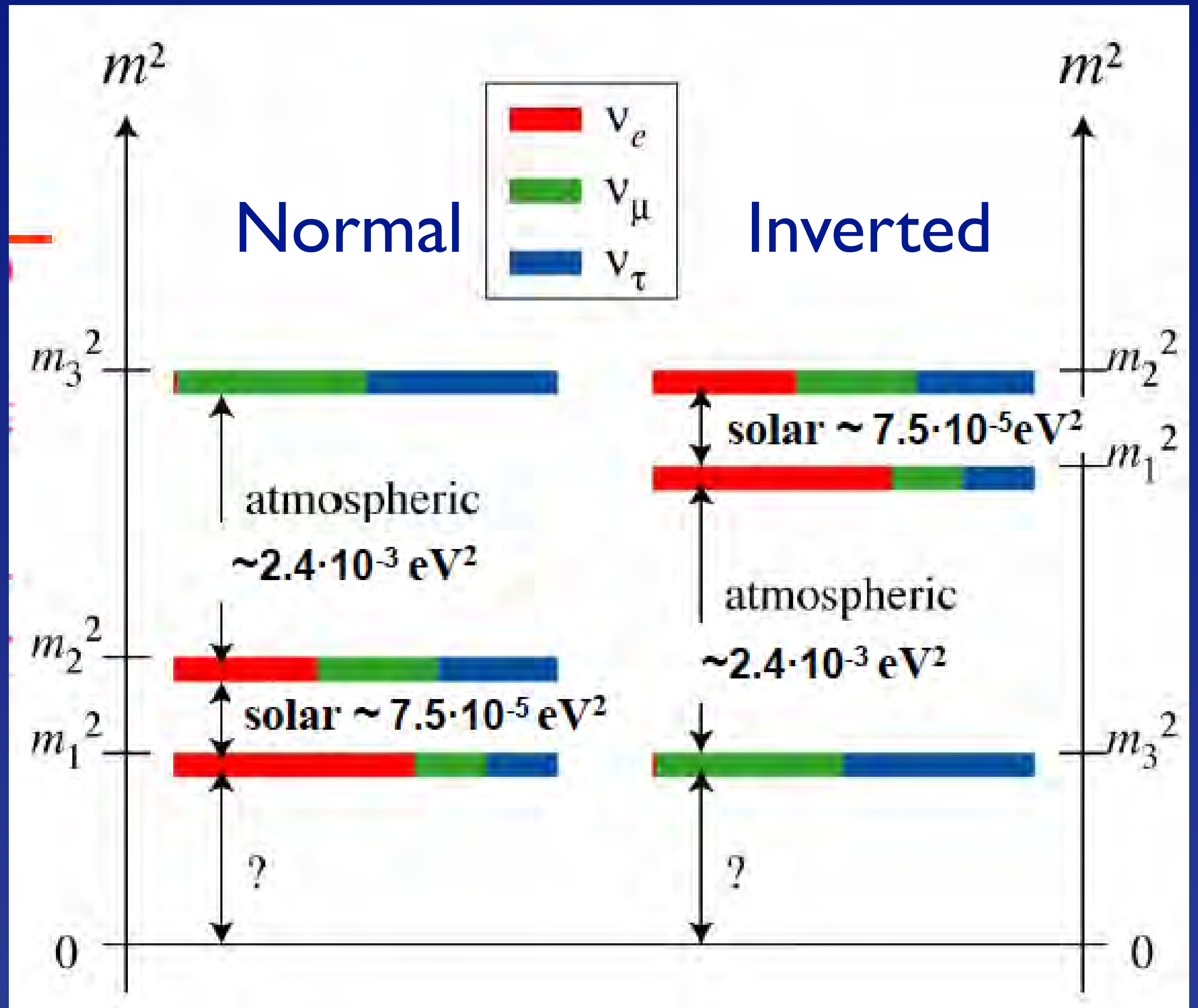

NOVA, T2K Ve appearance begin to hint normal hierarchy 


\section{Some outstanding questions in $\mathrm{V}$ physics}

CP Violation?

\section{T2K disfavors $0<\delta<\pi$ at $90 \% \mathrm{CL}$}

NOVA shows some sensitivity

$$
\begin{aligned}
& \text { Are neutrinos Majorana particles? } \\
& \text { Search for }(Z, A) \rightarrow(Z+2, A)+\text { ee: } \beta \beta_{0 v}
\end{aligned}
$$

Do 3 light neutrinos suffice?

Are there light sterile $v$ ?

Short baseline $\mathrm{V}$ experiments test for light steriles

\section{Might neutrinos decay?}

Can we detect the cosmic V background? 


\section{Tabletop precision experiments}

Electric dipole moment $d_{e}: \mathrm{CP} / \mathrm{T}$ violation

$$
\begin{gathered}
\left|d_{\mathrm{e}}\right|<8.7 \times 10^{-29} \mathrm{e} \cdot \mathrm{cm} \\
\text { ACME Collaboration, ThO } \\
\hline\left|d_{\mathrm{e}}\right|<1.3 \times 10^{-28} \mathrm{e} \cdot \mathrm{cm} \\
\text { NIST, trapped }{ }^{180} \mathrm{Hf}^{19 \mathrm{~F}^{+}} \\
\left(\underline{\text { SM phases: }} \mathrm{d}_{\mathrm{e}}<10^{-38} \mathrm{e} \cdot \mathrm{cm}\right)
\end{gathered}
$$




\section{Tabletop precision experiments}

(Anti)proton magnetic moments: CPT test

$$
\begin{gathered}
\mu_{\bar{p}}=-2.792847344 I(42) \mu_{N} \\
\text { vs. } \\
\mu_{p}=+2.792847350(9) \mu_{N}
\end{gathered}
$$

BASE Collaboration @CERN Antiproton Decelerator 


\section{Accelerator and magnet R\&D}

HE-LHC ( $x 2$ in energy) requires I5 T magnets:

$\mathrm{NbTi} \rightarrow \mathrm{Nb}_{3} \mathrm{Sn}$...

Nuclear \& particle physics consider e(p,A)

electron positron, circular or linear Higgs factory

high-energy lepton collider

More attention to neutrino factory 


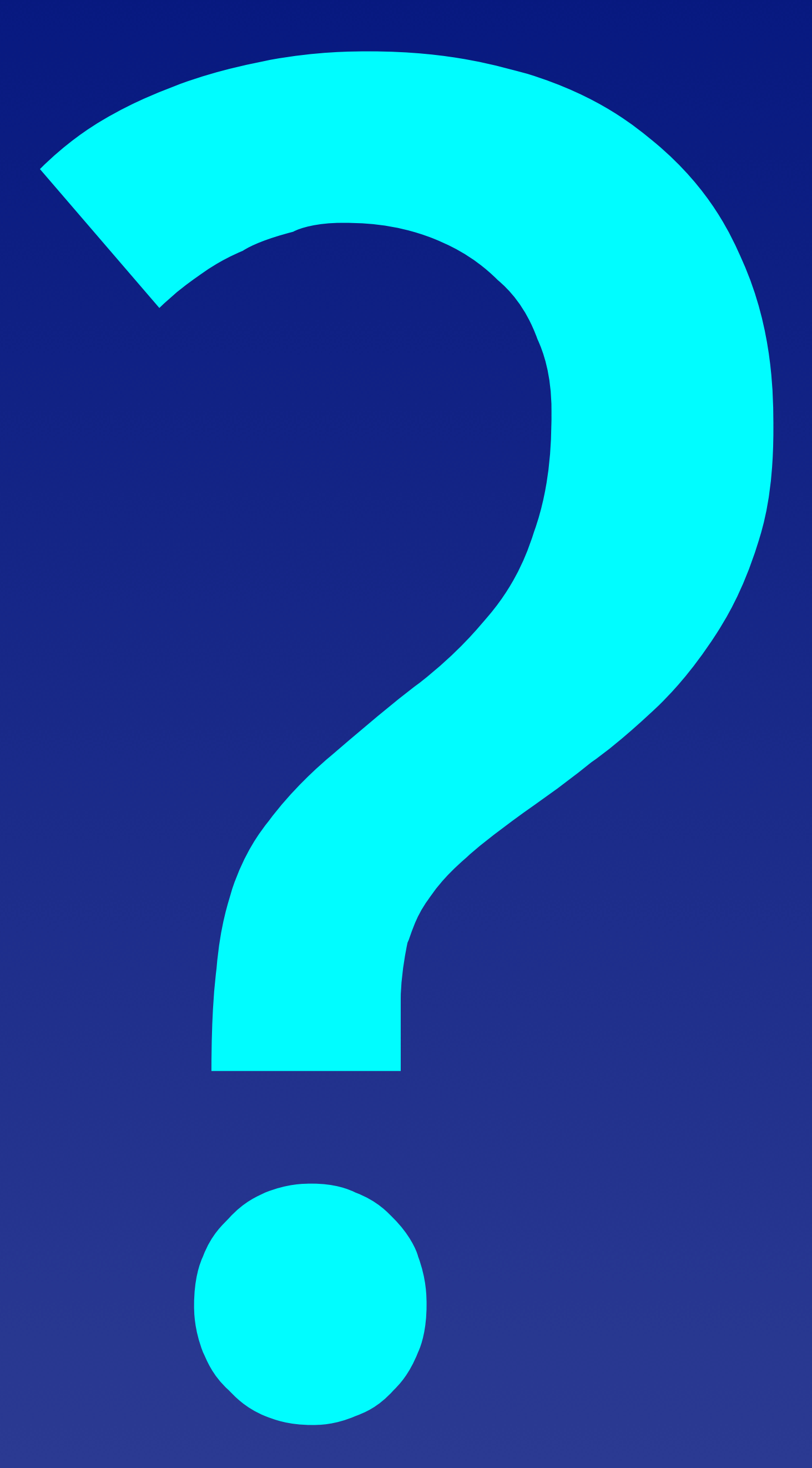




\section{Issues for the Future (Starting now!)}

I. There is a Higgs boson! Might there be several?

2. Does the Higgs boson regulate WW scattering?

3. Is the Higgs boson elementary or composite? How does it interact with itself? What triggers EWSB?

4. Does the Higgs boson give mass to fermions, or only to the weak bosons? What sets the masses and mixings of the quarks and leptons? (How) is fermion mass related to the electroweak scale?

5. Are there new flavor symmetries that give insights into fermion masses and mixings?

6. What stabilizes the Higgs-boson mass below I TeV? 


\section{Issues for the Future (Now!)}

7. Do the different $\mathrm{CC}$ behaviors of $\mathrm{LH}, \mathrm{RH}$ fermions reflect a fundamental asymmetry in nature's laws?

8. What will be the next symmetry we recognize? Are there additional heavy gauge bosons? Is nature supersymmetric? Is EW theory contained in a GUT?

9. Are all flavor-changing interactions governed by the standard-model Yukawa couplings? Does "minimal flavor violation" hold? If so, why? At what scale?

10. Are there additional sequential quark \& lepton generations? Or new exotic (vector-like) fermions?

II. What resolves the strong CP problem? 


\section{Issues for the Future (Now!)}

12. What are the dark matters? Any flavor structure?

13. Is EWSB an emergent phenomenon connected with strong dynamics? How would that alter our conception of unified theories of the strong, weak, and electromagnetic interactions?

14. Is EWSB related to gravity through extra spacetime dimensions?

15. What resolves the vacuum energy problem?

16. (When we understand the origin of EWSB), what lessons does EWSB hold for unified theories? ... for inflation? ... for dark energy? 


\section{Issues for the Future (Now!)}

17. What explains the baryon asymmetry of the universe? Are there new (CC) CP-violating phases?

18. Are there new flavor-preserving phases? What would observation, or more stringent limits, on electric-dipole moments imply for BSM theories?

19. (How) are quark-flavor dynamics and lepton-flavor dynamics related (beyond the gauge interactions)? 20. At what scale are the neutrino masses set? Do they speak to the TeV, unification, Planck scale, ...?

21 . Could our laws of nature be environmental?

22. How are we prisoners of conventional thinking? 